\title{
DESIGNING A MICROBIAL PROLYL PEPTIDASE DELIVERY SYSTEM FOR THE TREATMENT OF CELIAC DISEASE
}

\author{
A Thesis \\ presented to \\ the faculty of California Polytechnic State University, \\ San Luis Obispo
}

\author{
In Partial Fulfillment \\ of the Requirements for the Degree \\ Master of Science in Biological Sciences
}

by

Matthew J. Shurtleff

May 2009 
(C) 2008

Matthew J. Shurtleff

ALL RIGHTS RESERVED 


\section{COMMITTEE MEMBERSHIP}

TITLE: DESIGNING A MICROBIAL PROLYL PEPTIDASE DELIVERY SYSTEM

FOR THE TREATMENT OF CELIAC DISEASE

AUTHOR: $\quad$ Matthew J. Shurtleff

DATE SUBMITTED: May 2009

COMMITTE CHAIR: $\quad$ Michael Black, Ph.D

COMMITTE MEMBER: Christopher Kitts, Ph. D

COMMITEE MEMBER: $\quad$ Rafael Jimenez-Flores, Ph. D 


\section{ABSTRACT \\ DESIGNING A MICROBIAL PROLYL PEPTIDASE DELIVERY SYSTEM FOR THE TREATMENT OF CELIAC DISEASE \\ Matthew J. Shurtleff}

Celiac disease is an autoimmune enteropathy resulting from the ingestion of gluten and gluten-like proteins from wheat, barley and rye in afflicted individuals. Indigestible gluten-derived peptides rich in proline residues are known to be responsible for eliciting the inappropriate immune response characteristic of the disease. In this investigation, surface level expression of prolyl peptidase activity by genetically engineered probiotic lactobacilli was postulated to be a possible treatment for this disease. Plasmid-based reporter vectors were constructed utilizing a novel, homologybased cloning technique to assess the expression and localization signals from the S-layer protein gene of Lactobacillus acidophilus. These plasmids were mutated during construction due to toxicity associated with the cloned cassettes. The toxicity of the Slayer secretion and/or anchoring domains in E. coli was confirmed by cloning the fused components into an inducible expression system. When the prolyl peptidase, Xaa-Pro, from $L$. reuteri was incorporated into the S-layer expression cassette, the full-length protein was efficiently expressed but was not active, likely due to protein aggregation and inclusion body formation. Future research directions are discussed and a modified experimental design strategy is presented. This work provides a foundation for continued investigation into the feasibility of utilizing genetically engineered lactobacilli as a potential treatment strategy for celiac disease. 


\section{ACKNOWLEDGMENTS}

I thank Chris Kitts for sparking the fire.

I thank Rafael Jimenez-Flores for fueling the flame.

I thank Michael Black for shoving me into the blaze.

This work was generously supported by grants provided by the Office of Naval Research. 


\section{Table of Contents}

List of Figures....................................................................................................................................viii

List of Tables......................................................................................................................................ix

\section{CHAPTER}

1.0 Background ................................................................................................................1

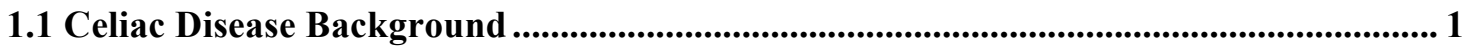

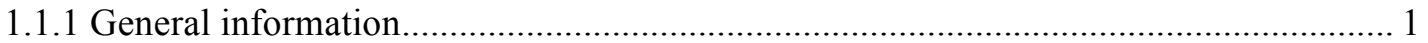

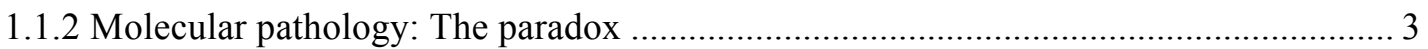

1.1.3 Gluten and related proteins: the source of toxicity in CD ........................................ 5

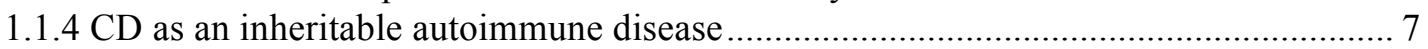

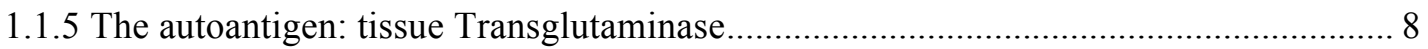

1.1.6 A case of molecular mistaken identity: A model for CD autoimmunity ....................... 9

1.1.7 The role of the innate immune system and intestinal permeability in CD ................... 11

1.1.8 Current and possible future treatments strategies to manage CD.............................. 12

1.2 Probiotic bacteria as enzyme delivery vehicles to manage CD........................................ 13

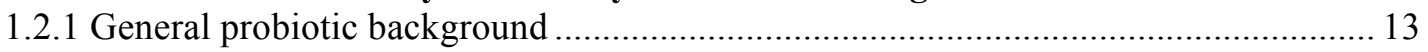

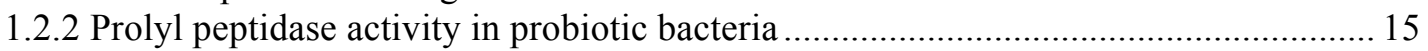

1.2.3 A novel treatment strategy for CD: genetically engineered probiotic bacteria ............. 16

\subsection{Construction of GFP Reporter Plasmids to assess the Efficiency of slpA}

Expression and Localization Signals..........................................................................................18

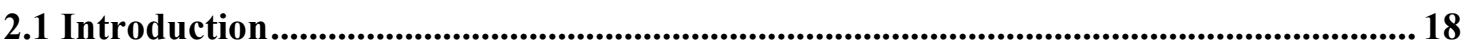

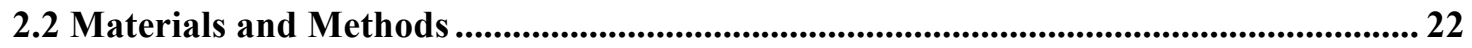

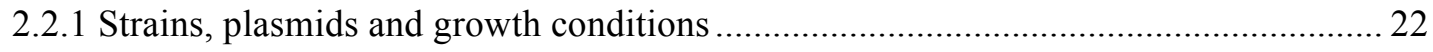

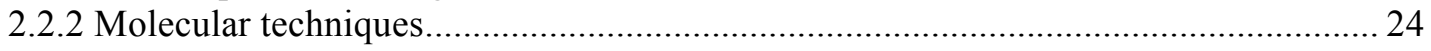

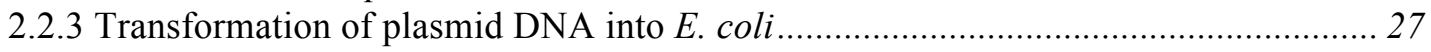

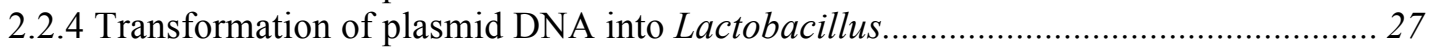

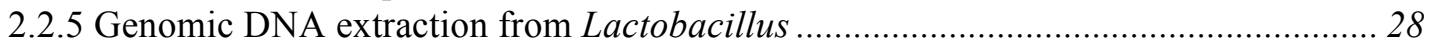

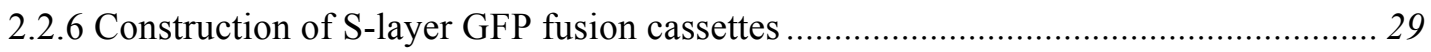

2.2.7 Construction of the pGKMCS shuttle plasmid...................................................... 30

2.2.8 Classical cloning of S-layer GFP cassettes into pNCKH113 and pGKMCS shuttle

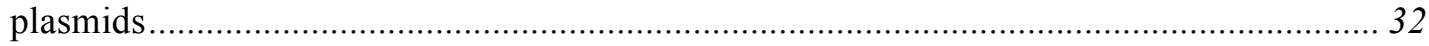

2.2.9 Digestion independent cloning of S-layer GFP cassettes into pGKMCS …................ 32

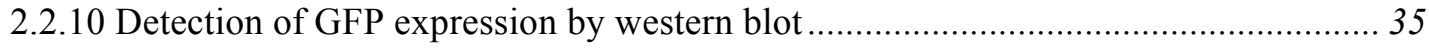

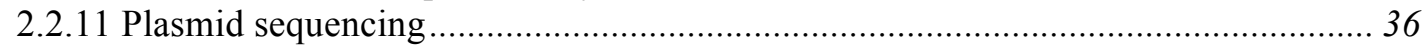

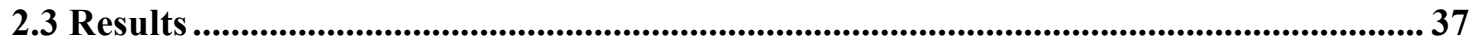

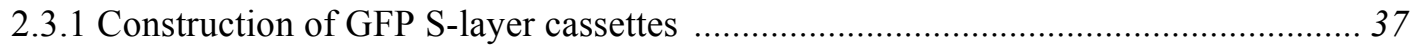

2.3.2 Transformation of shuttle vectors into E. coli, Lactobacillus acidophilus and L.

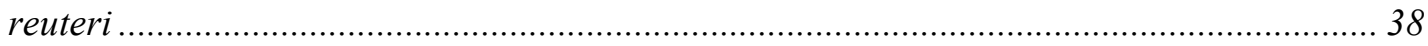

2.3.3 Cloning of S-layer GFP cassettes into pGKMCS and expression in E. coli .............. 39

2.3.4 Sequencing of the plasmids pGKMCS:PLGAT, pGKMCS:PLGT, pGKMCS:PGT. 43

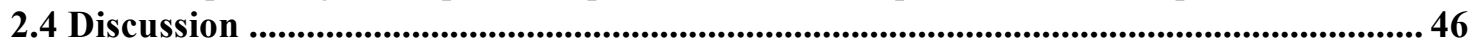


3.0 Construction of inducible plasmid systems for Lactobacillus and E. coli to determine localization of prolyl peptidase activity under control of the slp $A$

localization signals .....................................................................................................................53

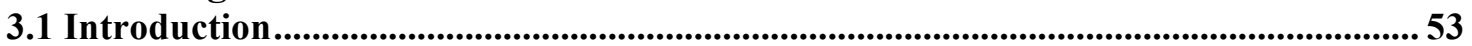

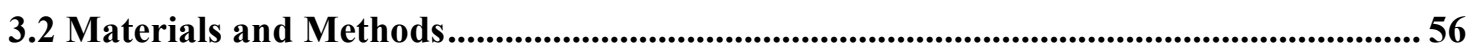

3.2.1 Strains, plasmids, growth conditions and molecular techniques...............................56

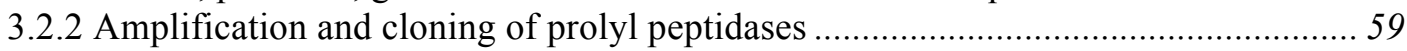

3.2.3 Construction of the erythromycin inducible expression vector pGK $\Delta$ Erm .............. 60

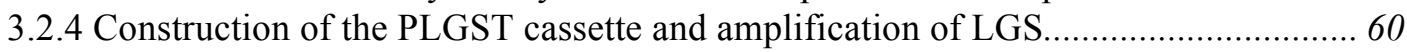

3.2.5 Construction of pGK $\Delta$ Erm:LGS, pGK $\Delta$ Erm:LXaaProS and pET30:LXaaProS....... 61

3.2.6 Western blot analysis of inducible GFP expression from pGK $\Delta$ Erm:LGS ............. 62

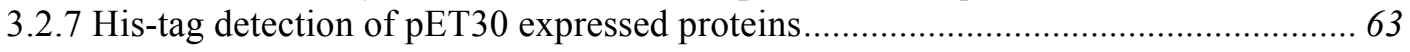

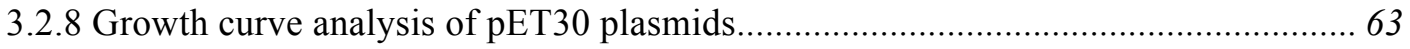

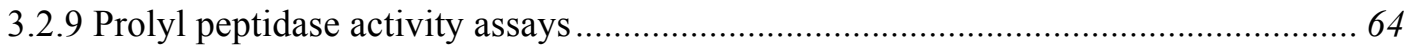

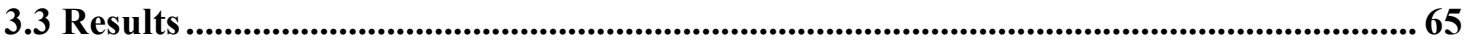

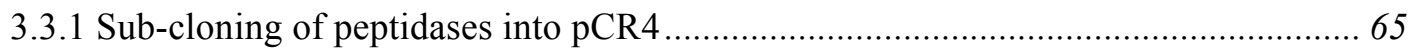

3.3.2 Characterization of the erythromycin inducibe plasmid pGK $\Delta$ Erm:LGS................ 66

3.3.3 Utilization of the pET30 expression system to determine the source of toxicity and

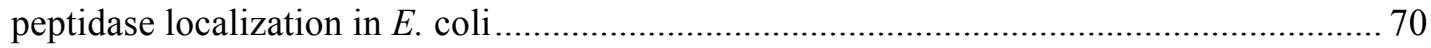

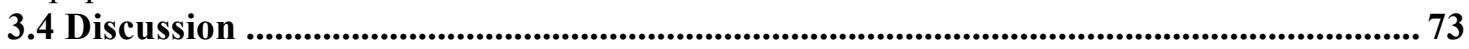

4.0 Concluding remarks and future research ........................................................................78

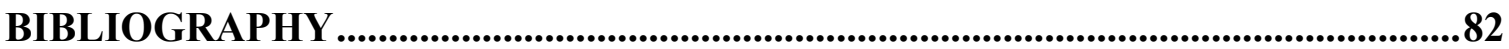




\section{List of Figures}

1-1 Molecular Pathogenesis of Celiac Disease ............................................................4

2-1 $\operatorname{slp} A$ expression and S-layer localization signals ..............................................21

2-2 Construction of the pGKMCS shuttle plasmid ..........................................................31

2-3 Digestion Independent Cloning (DIC) ..............................................................................34

2-4 Construction of the S-layer GFP cassettes ......................................................38

2-5 Microscopic analysis of $E$. coli and Lactobacillus strains transformed with Slayer GFP cassette-bearing plasmids ..................................................................42

2-6 GFP expression in E. coli containing S-layer GFPR cassettes ..............................43

3-1 Cloning of prolyl peptidases into the pCR4 TOPO-Blunt vector ..........................66

3-2 Erythromycin inducible GFP expression from the unstable pGKAErm:LGS plasmid ............................................................................................................................................68

3-3 Western blot analysis of inducible GFP expression in the stable pGKAErm:LGS plasmid.......................................................................................................69

3-4 pET30:LXS and pET30:LS Growth Curves....................................................70

3-5 Localization of LXS and LS in E. coli .....................................................71

3-6 Cellular morphology of induced and uninduced pET30:LS and pET30:LXS

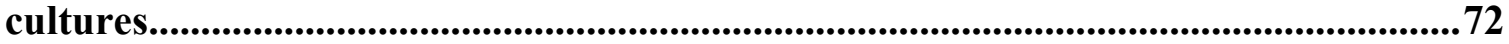




\section{List of Tables}

2-1 Plasmids used in this study (Chapter 2) .............................................................23

2-2 Primers used in this study (Chapter 2)................................................................26

2-3 S-layer GFP cassettes ...................................................................................................37

2-4 Sequencing of the inserts from pGK:PLGAT, pGK:PLGT and pGK:PGT

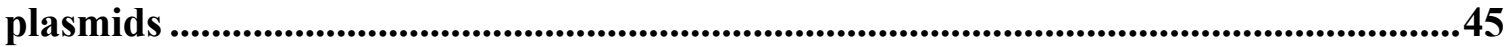

3-1 Plasmids used in this study (Chapter 3) ........................................................57

3-2 Primers used in this study (Chapter 3) .......................................................58 


\subsection{Background}

\subsection{Celiac Disease Background}

1.1.1 General information: Definition, Symptomology, Gross Pathology, Prevalence and Diagnosis

Celiac disease (coeliac disease, celiac sprue, CD) is an auto-immune enteropathy resulting from sensitivity to epitopes contained within gluten, the dominant protein portion of many common grains including wheat, barley and rye. Exposure to these proteins leads to inflammation and widespread atrophy of intestinal epithelia in individuals with $\mathrm{CD}$. Since these grains are ubiquitous in the average western diet, celiac disease greatly detracts from the quality of life enjoyed by those not suffering from CD.

Symptoms of CD are generally similar to other chronic gastrointestinal disorders such as Crohn's disease and ulcerative colitis, thus it is commonly misdiagnosed in both children and adults (Picarelli et .al., 2000; Corazza et al., 1996) . Common gastrointestinal symptoms include abdominal pain and cramping, flatulence, diarrhea, constipation and bloating. Chronic inflammation of cells lining the small intestine results in shortening and eventual complete destruction of villi. This leads to common extraintestinal manifestions of the disease, such as anemia, as well as other complications due to chronic malabsorption. CD primarily affects the proximal portion of the small intestine (the duodenum), but in some cases can extend into the ileum (Ruben et al., 1962). A subset of CD patients will develop a skin rash (dermatitis herpetiformis) in 
response to the ingestion of gluten that will resolve after administration of dapsone (the mechanism for this is not well understood). However, it should be noted that even if the only symptom of $\mathrm{CD}$ is dermatitis herpetiformis, the intestinal pathology is still manifested and these individuals remain at high risk for a number of celiac-associated conditions (Collin et al., 1996). The onset of CD symptoms varies within the population ranging from infancy to late adulthood. Diagnosis in young children is often very difficult due to the non-specific nature of the symptoms in this age group (failure to thrive, anorexia, pallor, musle atrophy), which may lead to misdiagnosis (Ciclitira, 2005). Approximately 50\% of diagnosed CD sufferers will not be diagnosed until adulthood, although the proportion of these individuals in which the onset of symptoms does not occur until adulthood as opposed to those whose symptoms were previously sub-clinical is not known. The prevalence of CD is approximately 1:100 among the Caucasian population, making it the most commonly diagnosed chronic gastrointestinal disorder (Ciclitira et al., 2005). Surveys of CD prevalence in other racial groups are sparse and poorly documented, likely because the Caucasian population is the most dependent on gluten containing grains as a primary nutritional source.

In cases in which $\mathrm{CD}$ is expected the patient will often be screened for the presence of auto-reactive antibodies (anti-endomysial and anti-tissue transglutaminase). Also, genetic screening can be useful as there is a strong genetic association with $\mathrm{CD}$ in the class II major histocompatibility complex (MHC-class II) HLA-DQ locus, with $\sim 98 \%$ of CD cases having either the DQ2 alleles ( $\sim 90 \%)$ or the DQ-8 alleles ( $\sim 8 \%)$ (Sollid \& Thorsby, 1993). Although both screening methods are useful in either eliminating CD as 
a possibility or narrowing down the focus of the physician, ultimately $\mathrm{CD}$ diagnosis must be confirmed by intestinal biopsy.

\subsubsection{Molecular pathology: The paradox}

A feature of $\mathrm{CD}$ molecular pathogenesis that makes the disease relatively unusual among autoimmune disorders is that the immune response, though directed at host tissues, is actually initiated and propagated by foreign antigens derived from the partial digestion of $\alpha$-gliadin, a protein within gluten. Only recently, after the discovery of the epitope structures recognized by auto-reactive antibodies and the subsequent elucidation of the immunodominant gliadin-derived peptides, has a model for the initiation and propagation of the humoral autoimmune response emerged (Figure 1-1). In order to understand how this paradox of a foreign antigen capable of eliciting an autoimmune response can be resolved, some consideration must first lent to major players involved in the complex molecular pathology of this hereditary autoimmune disorder. 


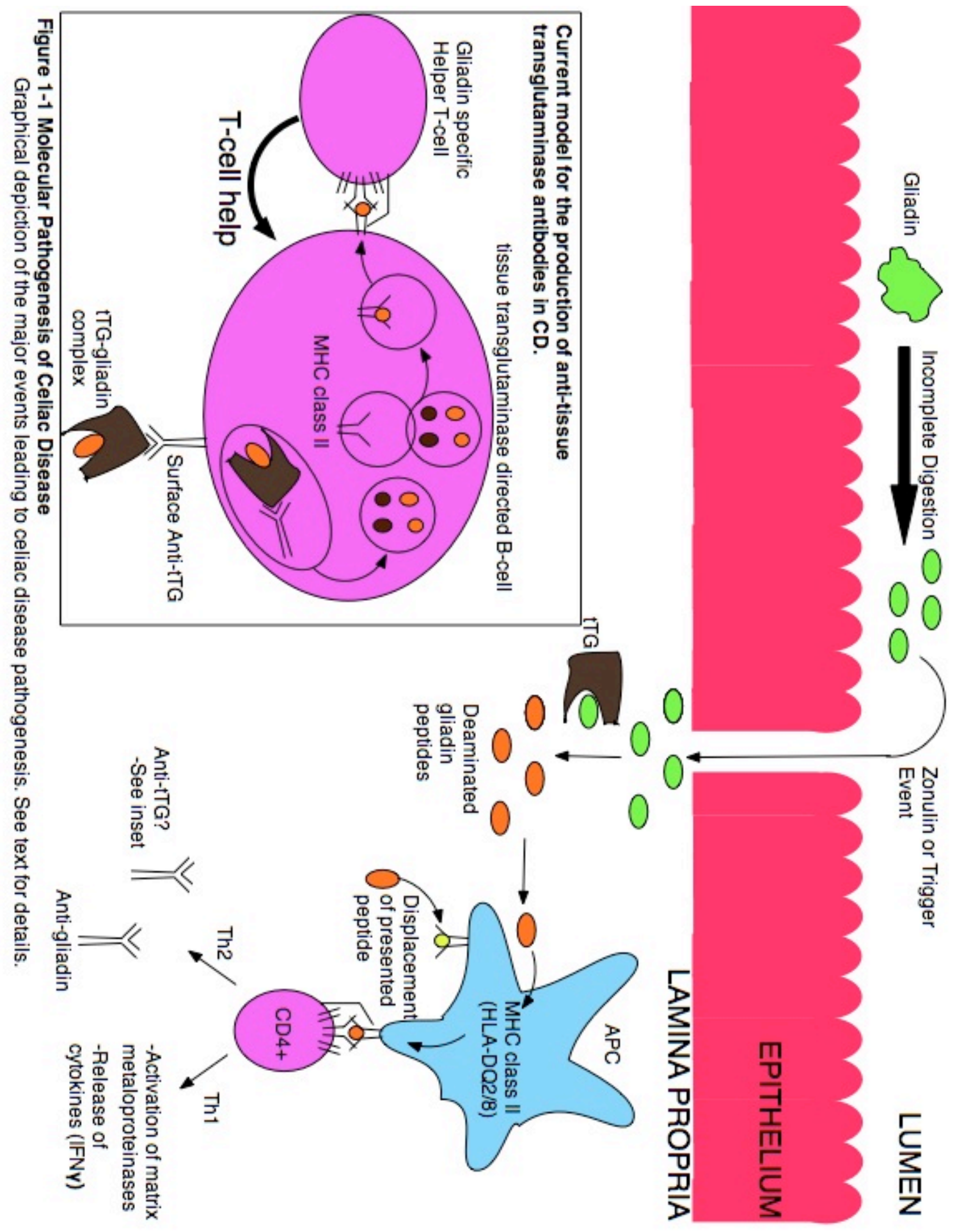




\subsubsection{Gluten and related proteins: the source of toxicity in $\mathrm{CD}$}

Gluten is the dominant proteinaceous portion of wheat. Closely related protein elements are present in barley and rye grains that are also capable of eliciting the immune response characteristic of celiac disease. For simplicity, only gluten will be discussed here, as the overall mechanism of pathogenesis is the same between grains with only the names differing. Gluten can be split into two general types of proteins, the glutenins (low molecular weight proteins) and the gliadins (high molecular weight proteins, denoted $\alpha$ $\gamma)$. While both glutenins and gliadins contain epitope structures capable of initiating an immune response, the dominant peptides responsible for eliciting immune response (immunodominant) are derived from $\alpha$-gliadin (Shan et al., 2002).

Two short peptide epitope structures, PFPQPLPY and PQPQLPYPQ have been identified as the primary epitopes in CD pathogenesis. The particular importance of these two specific epitopes is evidenced by the observation that gluten specific T-cells from nearly all CD patients respond to one or both of these peptides (Arentz-Hansen et al., 2000). Furthermore, when these two peptides are administered to individuals that have been on a strict gluten exclusion diet, T-cells responding to these two peptides are the first to be detected in the peripheral blood stream (Anderson et al., 2000). Seminal work by Lu Shan of the Khosla research group proposed that a single 33 amino acid peptide might be the primary factor initiating an autoimmune response. This peptide, along with another 18 amino acid peptide, have been designated as the immunodominant CD peptides. Challenge of gut-derived T-cell lines from nearly all $\mathrm{CD}$ patients tested with this 33-mer resulted in induction of these cells, giving support for the hypothesis that this 
toxic peptide is the immunodominant peptide in the majority of $\mathrm{CD}$ patients. This peptide also contains 6 partially overlapping epitope structures and is highly degradation resistant due to the large number of proline residues (Shan et al., 2002).

Proline is unique among amino acids in that it has its side chain wrapped around and bonded to the nitrogen atom involved in peptide bonding. This unusual bonding scheme results in tight kinks being formed in the primary structure of peptides containing proline. If there are a high number of proline residues the peptide will twist back upon itself, creating a proteolysis resistant structure that cannot be degraded by common digestive enzymes in the human gastrointestinal tract. The 33-mer peptide has a half-life of at least 20 hours when subjected to brush border membrane digestive enzymes, with the relatively poorly expressed peptidases dipeptidyl carboxypeptidase I and dipeptidyl peptidase IV (DPPIV) identified as the rate limiting factors in breakdown of this troublesome peptide (Shan et al., 2002, Hausch et al., 2002). The facts that this peptide contains immunoreactive epitope structures and a high proline content conferring digestive resistance are each contributing factors to toxicity. However, this only suggests that an immune response would be targeted to gliadin, resulting in the generation of antigliadin antibodies. The question that remains is: How can this foreign peptide result in an autoimmune response targeted against a structurally distinct and ubiquitously expressed host enzyme (tissue transglutaminase)? An answer to this question can only be arrived at after a thorough discussion of how CD is fundamentally a genetically-based disorder. 
1.1.4 $\mathrm{CD}$ as an inheritable autoimmune disease

Clear evidence of $\mathrm{CD}$ as an inheritable disorder has been evidenced by sibling and twin studies. The most recent large-scale population study showed a high concordance rate (70-75\%) of CD between monozygotic twins (Greco, 2002). As previously mentioned, a strong genetic association exists between CD and the HLA-DQ genetic locus with the vast majority of CD sufferers possessing the HLA-DQ2 ( 90\%) or HLADQ8 ( 8\%) genotype. The HLA-DQ locus encodes MHC class II proteins responsible for presentation of foreign proteins or peptides. When a foreign protein is encountered, it is endocytosed by an antigen presenting cell (APC), degraded and processed in the late endosome and loaded into the HLA-DQ heterodimer. Once loaded, the HLA-DQ+peptide complex is delivered to the plasma membrane of the APC where it can be recognized by the corresponding helper T-cell. Once the helper T-cell recognizes the specific antigen to which it is programmed to respond, the cell will be activated and begin producing and secreting cytokines. In CD, a TH1 response is mounted involving the release of IFN- $\gamma$, resulting in inflammation and activation of the innate immune response and eventually culminating in cellular destruction. As will be discussed later, a TH2 response also plays an important role in $\mathrm{CD}$ pathology by activating B-cells to produce anti-gliadin and antitissue transglutaminase antibodies.

More than a simple genetic correlation, the HLA-DQ2 association appears to have a functional role in $\mathrm{CD}$. This version of the MHC-class II proteins has a strong predilection for negatively charged molecules. However, immunodominant peptides do not have an overall negative charge. How can sense be made of this apparent 
incompatibility? To address this question a discussion of the functional role of the CD autoantigen, tissue transglutaminase is warranted.

\subsubsection{The autoantigen: tissue transglutaminase}

In 1997 the autoantigen for CD was identified as tissue transglutaminase (tTG) (Dietrich et al., 1997). Tissue transglutaminase is a ubiquitously expressed enzyme whose primary functional role appears to be the cross-linking of fibronectin proteins during clot formation. Under the slightly acidic conditions present in the small intestine however, the enzymatic activity of tTG switches from cross-linking of glutamine to lysine residues (transamidation) to deamidation (Fleckenstein et al., 2002). Deamidation of glutamine residues results in the conversion of glutamine to glutamic acid, a negatively charged amino acid. tTG has an extremely high affinity for the CD associated immunogenic peptides which is strong enough to displace the enzymes primary substrate fibronectin (Xia et al., 2005). Interestingly, it has been found that partial deamidation of glutamine residues present in immunogenic peptides is required to initiate an immune response whereas no deamidation or complete deamidation of the same peptides did not result in the activation of gut derived T-cells in patients with CD (Molberg et al., 1998). This further underlines the essential role of this host protein in the pathogenesis of CD. Furthermore, the very close association between immunogenic peptides and tTG is also the foundation for the current model describing how these foreign peptides can result in an autoimmune response directed against the $\mathrm{CD}$ autoantigen, tTG. 


\subsubsection{A case of molecular mistaken identify: A model for CD autoimmunity}

The immune system contains three primary types of APCs; dendritic cells, macrophages and B-cells. The first two cell types are the basis of the adaptive immune response, constantly sampling the environment and presenting a wide range of potential antigens on their surfaces to be recognized by helper T-cells. Once the helper T-cell recognizes an antigen presented by either a macrophage or dendritic cell, a TH1 response will be generated. In a TH1 response, the helper T-cell will begin secreting cytokines (IFN $\gamma$ among others) that promote inflammation and lead to the recruitment of other cells involved in the immune response. Also during this response, matrix metalloproteinases are activated leading to widespread tissue damage. It is likely that the TH1 response is greatly amplified in $\mathrm{CD}$ due to the extremely high affinity of the deaminated immunodominant peptides for the HLA-DQ2 proteins. This affinity is so strong that, immediately following the initial influx of gliadin into the intestinal sub-mucosa, these peptides are able to displace those antigens that are already being presented by APCs in the lamina propria (Xia et al., 2005).

Helper T-cells that recognize antigens presented by B-cells are responsible for mounting the $\mathrm{TH} 2$ response. A $\mathrm{TH} 2$ response leads to the activation of B-cells resulting in the production of antibodies. B-cells are unique among antigen presenting cells in that they can only present the specific antigen for which they are programmed to recognize. This occurs through the binding of the antigen to surface immunoglobulin, this complex is then endocytosed, processed in much the same was as other APCs and presented on its surface for identification by helper T cells. This leads to activation of helper T-cells leading to the $\mathrm{TH} 2$ response, resulting in the release of cytokines that signal B-cells to 
begin producing antibodies. While there are likely to be many auto-reactive B-cells circulating (left over from the early maturation of the immune system) these will generally not result in auto-antibody production, because there is no corresponding helper T-cell to signal antibody production. These auto-reactive "rogue" B-cells along with the tight interaction between tTG and the gliadin-derivded immunodominant peptides (ID) form the crux of the current model for the molecular pathogenesis of $\mathrm{CD}$ and the production of anti-tTG antibodies.

B-cells with anti-tTG surface immunoglobulin bind to tTG interacting with ID peptides, the tTG-ID complex is then endocytosed, processed and the antigens are presented on the cell surface. If the processed antigen is derived from tTG, there will be no corresponding helper T-cell dependent activation and no antibody will be produced. However, if the presented antigens are derived from the tTG bound ID then ID specific helper T-cells will signal the antigen presenting B-cell to produce the antibodies it is programmed to release, anti-tTG. Again the extremely high affinity of deaminated ID peptides for the HLA-DQ2 proteins comes into focus and plays an essential role in this model for auto-antibody production. The affinity of ID for these proteins is so high that they can displace other peptides that are already bound in the late endosome, thus essentially ensuring that the deaminated peptides, rather than a fragment from tTG will be the presented antigen (Xia et al., 2005). The exact role of anti-tTG in the intestinal pathology of CD remains unclear and controversial. It is likely that the inflammatory response generated from $\mathrm{TH} 1$ activation is the primary contributor to tissue damage and symptomology, with the production of anti-tTG antibodies playing a lesser role in intestinal pathology. 
1.1.7 The role of the innate immune system and intestinal permeability in $\mathrm{CD}$

The innate immune response had previously been thought to play an insignificant role in CD pathogenesis. Recent evidence however, contradicts this viewpoint and suggests that components of the innate immune response are necessary for the initial influx of gliadin peptides into the intestinal sub-mucosa.

Zonulin is a protein that is commonly released in response to bacterial infection that results in the phosporylation of proteins that maintain tight junction integrity, thus leading to increased intestinal permeability. Gliadin exposure to normal and CD intestinal mucosa also activates the zonulin signaling pathway (Drago et al., 2006). Recent work has further clarified the upstream events leading to zonulin release. In this model, gliadin peptides bind to the CXCR3 chemokine receptor in mucosal cells leading to recruitment of the MyD88 adaptor protein which then signals the release of zonulin leading to increased intestinal permeability (Lammers et al., 2008). This role of the innate immune response in promoting the invasion of gliadin across the intestinal barrier and into the lamina propryia where it can interact with members of the adaptive immune system appears to be an essential initial step in the pathogenesis of CD. Furthermore, it has been postulated that MyD88 dependent zonulin release may be an important contributor to a number of other autoimmune diseases, some of which show comorbidity with CD (Watts et al., 2005; Thomas et al., 2006). 


\subsubsection{Current and possible future treatments to manage CD}

The only current treatment strategy available to manage CD is the gluten-free diet. Unfortunately, it is extremely difficult to avoid gluten entirely in a modern western diet that is strongly founded on grains. Individuals with CD can rarely eat out at restaurants as many common cooking components contain enough gluten to trigger an immune response. In many cases only very small, accidental exposures can be enough to lead to a full-scale immune response and the resulting symptoms. Furthermore, there is a very high degree of non-compliance among CD patients on the gluten-free diet as many individuals find the gluten-free foods unsavory, or are unaware of the presence of gluten in their food. Indeed, gluten can be found in unexpected sources such as vitamins and soy sauce. While the symptoms of CD may be tolerable or even sub-clinical in some individuals, the associated conditions and long-term effects of unmanaged CD can be severe or even fatal. These include; skin disorders, ulcerative colitis, lymphocytic colitis, liver disease, thyroid disease, anemia, diabetes, bone metabolism, malignancy and even neurological and psychological disturbances (Ciclitira et al., 2005). Management of celiac disease through the gluten free diet is critical to limiting and preventing complications, especially life threatening complications such as gastrointestinal lymphoma and carcinoma (Corrao et al., 2001).

Recently a new treatment strategy based upon the possibility of enzyme therapy using prolyl endopeptidases derived from bacteria or fungi has been proposed (Shan et al. 2004, Stepniak et al. 2006). These enzymes break down the immunodominant peptides by hydrolyzing proline-Xaa bonds. Unfortunately, these enzymes would have to be delivered as purified enzyme, since none of the organisms from which these enzymes 
have been isolated can colonize the small intestine. As the human digestive tract is in fact a biological system, the protein expression profile is an amalgam of both human digestive enzymes as well as the multitude of microbial digestive enzymes produced by the bacteria that inhabit the intestines. Armed with this knowledge, a continued exploration into the prospects for a potential microbial-based treatment is justified.

\subsection{Probiotic bacteria as enzyme delivery vehicles to manage CD}

\subsubsection{General probiotics background}

Bacteria that stabilize the intestinal tract and promote healthy digestion are termed probiotic. Although the term probiotic is relatively new the idea of beneficial bacteria is as old as the study of our defenses against harmful bacteria. The term probiotic was coined in 1965 to describe the ability of certain microorganisms to promote growth and maturation in some animals. This definition was refined several times over the years and a more modern definition of the term has developed to more specifically describe these bacteria. "Probiotics is a microbial dietary adjuvant that beneficially affects the host physiology by modulating mucosal and systemic immunity as well as improving nutritional and microbial balance in the intestinal tract" (Naidu et al., 1999). This definition however, may require further modification as researchers are discovering beneficial effects of bacterial colonization throughout the body. Classically, probiotic bacteria are primarily members of the lactic acid family of bacteria (LAB). LABs are Gram positive, non-spore forming bacteria that produce lactic acid from the fermentation 
of sugars. Lactobacillus is the largest genus of LABs consisting of more than 50 species (Stiles \& Holzapfel,1997; Tannock, 2004). Lactobacilli are important for the production of foodstuffs. Most notable among these organisms are Lactobacillus delbrueckii ssp. bulgaricus and Lactobacillus sanfranciscensis used in the commercial production of yogurt and sourdough bread, respectively. While their primary role in food production is the fermentation of sugars to lactic acid, non-commercially important LABs are also responsible for producing a wide variety of compounds beneficial to humans, including anti-microbial peptides, exopolysaccharides and other metabolites (Ross, Morgan \& Hill, 2002).

An important role of probiotic bacteria in the human intestinal tract is the regulation of intestinal homeostasis. This involves protecting the mucosal epithelium from pathogens, delivery of regulatory signals to the immune system and neuromuscular system, as well as inhibition of pathogenic bacteria through nutrient competition, binding translocation and production of anti-microbial peptides (Shanahan, 2004). The genus Lactobacillus has been shown to be effective in the treatment and prevention of gastrointestinal disorders such as infectious diarrhea and inflammatory bowel disease (Saavedra, 2000; Servin, 2004; Shanahan, 2004).

Probiotic bacteria must be able to bind to intestinal surfaces in order to exert their beneficial effects. Binding to the mucosal epithelia of the intestinal tract by lactic acid bacteria is not fully understood. Two probiotic species, Lactobacillus reuteri and Bifidobacterium bifidum bind competitively to the same glycolipid carbohydrate moieties in the intestinal tract that are recognized adhesion factors for enteropathogenic bacteria (Mukai et al. 2004). Importantly, several LABs are able to survive and colonize the small 
intestine, the primary site of gastrointestinal pathology (Marteau et al. 1997). Notably, a strain of Lactobacillus reuteri can colonize the proximal portion of the small intestine, the site of celiac pathology (Valeur et al., 2004)

Another potential benefit of gastrointestinal colonization by probiotic bacteria is supplementation of enzymes in which the human intestinal tract is deficient. A wellknown example of this is the ability of lactose intolerant individuals to be able to ingest lactose without negative symptoms after a treatment regiment of probiotic bacteria. In this case, the individual is deficient in a functional $\beta$-galactosidase, which is provided by the LABs that have colonized the intestines during treatment (De Vrese et al., 2001). The lactose intolerance example makes one question what other enzymes probiotic bacteria may express that could be beneficial to humans.

\subsubsection{Prolyl peptidase activity in probiotic bacteria}

Prolyl peptidase activity is conspicuously absent from the human digestive tract, with only the poorly expressed Dipeptidyl peptidase IV able to act on proline-rich substrates. These enzymes however can be found throughout the microbial community. Prolyl peptidase activity can be found in abundance throughout members of the genus Lactobacillus. Due to their inability to synthesize many amino acids, LABs are dependent upon extracting their amino acid requirements from the environment. For this reason LABs (especially lactobacilli) maintain an extremely complex and diverse proteolytic system. Prolyl peptidase activity has already been characterized in several species in the 
LAB family including, Lactobacillus helveticus, Lactobacillus sakei, Lactobacillus reuteri and Lactococcus lactis (Degraeve et al., 2003; Sanz et al., 2001; Rollan \& Font de Valdez, 2001; Xu et al. 2002). Furthermore, LABs are capable of detoxifying glutenderived peptides during food processing (Rizzelo et al. 2007, De Angelis et al. 2006).

1.2.3 A novel treatment strategy for CD: genetically engineered probiotic bacteria Several of the bacteria discussed in the previous section that possess prolyl peptidases are able to colonize the intestines, including the small intestine. A bacterial strain that can colonize the small intestine and constitutively express and secrete prolyl peptidase enzymes at a high level would effectively supplement the area with enzymes capable of detoxifying gluten. This approach to treatment probably will not allow individuals with $\mathrm{CD}$ to eat a loaf of bread, but it may allow for tolerance of small amounts of gluten and could therefore protect against accidental and unforeseen exposure. Furthermore, if a probiotic bacterium such as those described in previous sections were used, the individual would not only alleviate the CD symptoms due to prolyl peptidase activity, but also acquire additional health benefits attributed to intestinal colonization by probiotics. Unfortunately, no probiotic microorganism yet studied secretes prolyl peptidase enzymes beyond the cytoplasm. However, due to the advancements in molecular biology technology, it may now be possible to engineer strains capable of surface expression of prolyl peptidases. This hypothetical strain would be able to colonize the small intestine and express prolyl peptidases on the surface of the cell, thus effectively localizing enzyme activity at the site of celiac pathology. The 
remainder of this document describes the initial research undertaken to achieve this single goal, to provide the first non-dietary treatment option for celiac disease. 


\subsection{Construction of GFP Reporter Plasmids to Assess the Efficiency of $\operatorname{sip} A$ Expression and Localization Signals}

\subsection{Introduction}

The microbial S-layer is a two-dimensional crystalline matrix that completely covers some members of the Eubacteria, and nearly all members of the Archaea. This specialized cellular structure is comprised of a single (with few exceptions) protein that is able to self-assemble, in a purely thermodynamically driven process, into a highly ordered crystalline matrix at the cell surface.

One extensively studied S-layer protein is the SlpA protein of Lactobacillus acidophilus. The slpA gene encodes the open reading frame for the 444 amino acid SlpA protein as well as two putative promoter sequences and a transcription termination sequence (Boot et al., 1993; Boot et al., 1996). The slpA gene resides on a $\sim 6 \mathrm{~kb}$ chromosomal inverted repeat which contains both the $\operatorname{slp} A$ ORF as well as the very closely related $\operatorname{slp} B$ ORF. The $\operatorname{slp} B$ ORF does not have a promoter sequence and thus cannot be expressed unless a chromosomal inversion event occurs that places the $\operatorname{sip} B$ ORF behind the $\operatorname{slp} A$ promoter, the mechanism responsible for this inversion has not yet been identified but has been suggested to be mediated by a protein similar to the Din invertase of E. coli (Boot et al., 1996).

The slpA gene contains two putative promoter sequences upstream of the ORF (Figure 2-1a), however only one of these promoters (the promoter nearest to the ORF) is 
used to drive expression of SlpA (Boot et al., 1996). It is possible however that the upstream promoter may play a role in expression under growth conditions not yet tested. This promoter combined with the extensive 5' mRNA untranslated region (UTR) has been reported to be one of the strongest expression contexts in Gram positive bacteria. At any given time the SlpA protein can constitute as much as $10-15 \%$ of the total cellular protein (Boot et al. 1996). The extremely high level of expression is the result of two elements. 1) The slpA promoter is among the most efficient in lactic acid bacteria (Yeh et al., 2008). 2) The 5' untranslated region (UTR) folds into a highly stable loop structure protecting the mRNA from $5^{\prime} \rightarrow 3^{\prime}$ degradation (Boot et al., 1996). Deletion of the 5 'UTR results in at least a 2-fold drop in expression (Boot et al., 1996, Narita et al., 2006).

The SlpA protein is composed of three components, a N-terminal leader sequence that directs secretion, a crystallizing domain and a C-terminal anchoring domain. For the purposes of this investigation the leader and anchor regions will be talked about in detail. The leader sequence comprises the first 24 amino acids of the ORF and contains a leader sequence (MKKLNR) for general secretion via the SecII secretion pathway (Figure 21b). The SecII pathway is a generalized pathway for secretion of proteins in bacteria and has a well-conserved consensus sequence that directs cleavage of the pro-protein during secretion. The predicted cleavage site for the slpA pro-protein is at amino acid position 24 and is consistent with the molecular weight $(40.4 \mathrm{kD})$ of the mature SlpA protein (Boot et al. 1993). The C-terminus anchoring domain is comprised of 2 tandem repeats, Sac1 and Sac2 (Smit \& Pouwels, 2002). These two sequences are primarily comprised of basic and aromatic amino acids and share 26\% identity (Figure 2-1c). Evidence suggests that the 
anchoring domain binds to peptidoglycan associated teichoic acids and that Sac1 alone is necessary and sufficient for binding (Smit \& Pouwels, 2002).

The purpose of this investigation is to address the possibility of using S-layer derived signals to drive expression and localization of a reporter protein, green fluorescence protein (GFP). In order to achieve this goal, a plasmid-based expression system was utilized and the efficiency of expression and functionality of localization elements were assessed by fluorescence microscopy and western blot analysis. 


\section{a) sIpA promoter sequences and 5' UTR}

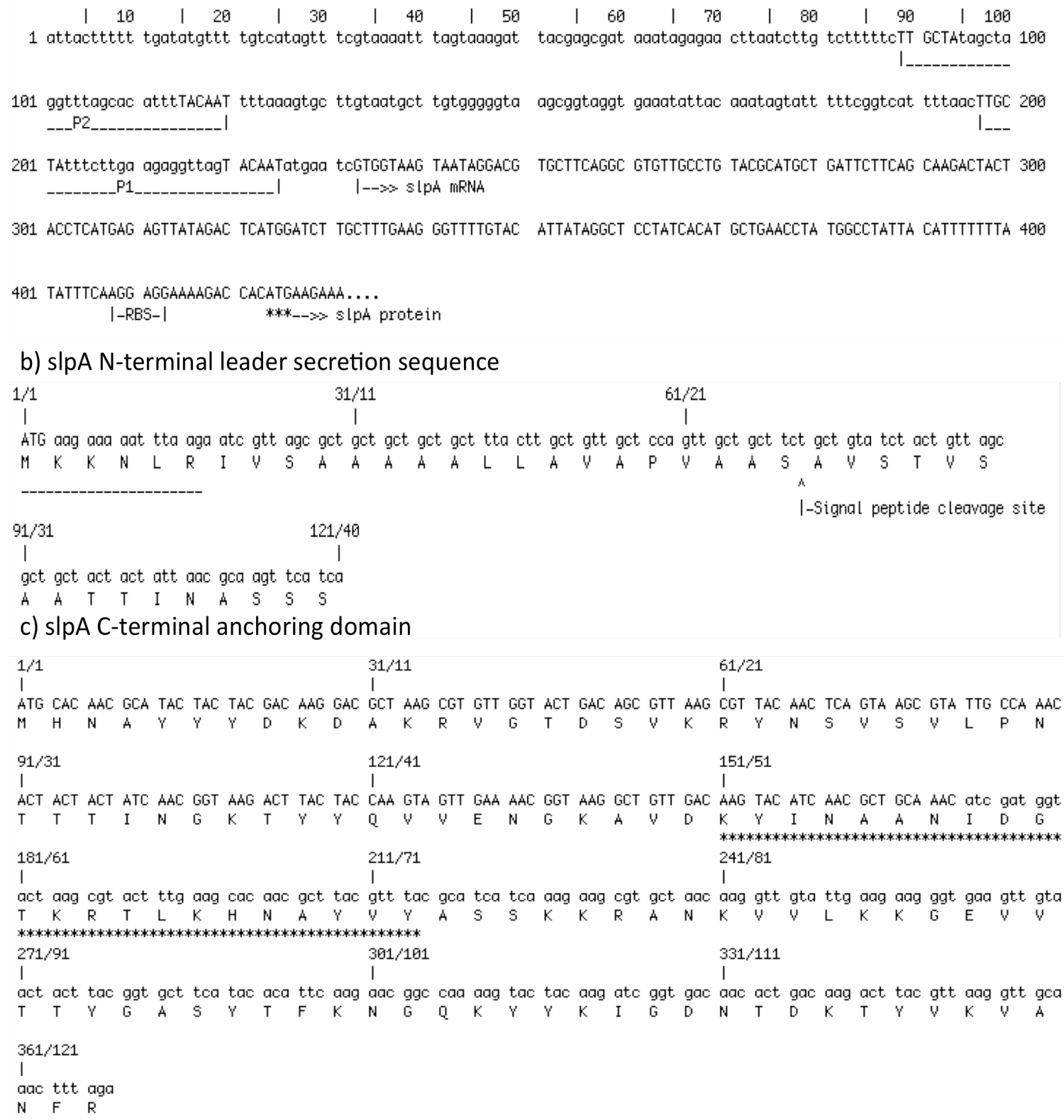

\section{b) slpA N-terminal leader secretion sequence}

Figure 2-1 $\operatorname{slp} A$ expression and S-layer localization signals.

a) The $\operatorname{slp} A$ promoter sequence including the putative promoter sequences P1 and P2 (-10 and -35 consensus sequences in caps) and the 5' UTR region (in caps, $* * *=s \operatorname{lp} A$ start codon).

b) The $\operatorname{slp} A$ leader sequence including the secretion signal (underlined) and the predicted cleavage site for the immature protein.

c) The $\operatorname{slp} A$ S-layer anchoring domain with Sac1 (nucleotides in caps) and Sac2 (nucleotides in lower case) tandem repeats. The sequence underscored by asterisks indicates the region likely involved in murein hydrolase activity. (Acosta et. al., 2008 - See discussion). 


\subsection{Materials and Methods}

2.2.1 Strains, plasmids and growth conditions

Several E. coli strains were utilized in this study including: TOP10 (Invitrogen), T-1 (Zymo research), JM109, DH5 $\alpha$ and NEB Turbo (New England Bio Labs, NEB). Lactobacillus acidophilus NCFM was provided by the Enviromental Biotechnology Institute, Cal Poly SLO and Lactobacillus reuteri ATCC 23272 was provided by the Dairy Products Technology Center, Cal Poly SLO. E. coli was propagated on LuriaBertani (LB) plates and antibiotic resistant strains were selected for with erythromycin $(250 \mathrm{ug} / \mathrm{mL})$, chloramphenicol $(15 \mathrm{ug} / \mathrm{mL})$ and ampicillin $(100 \mathrm{ug} / \mathrm{mL})$ as required. Lactobacilli were grown in Man-Rogosa-Sharpe (MRS) media and plasmid-bearing strains were selected for using erythromycin $(5 \mathrm{ug} / \mathrm{mL})$.

The three shuttle plasmids pGK12, pGKNucMCS and pNCKH113 were obtained from T. Klaenhammer (North Carolina State University), The American Type Culture Collection (ATCC- Bethesda, Maryland) and G. Tannock (University of Otago) respectively. The plasmid pMB293 (the source of green fluorescent protein) was built previously (M. Black) and was acquired from the laboratory collection. 


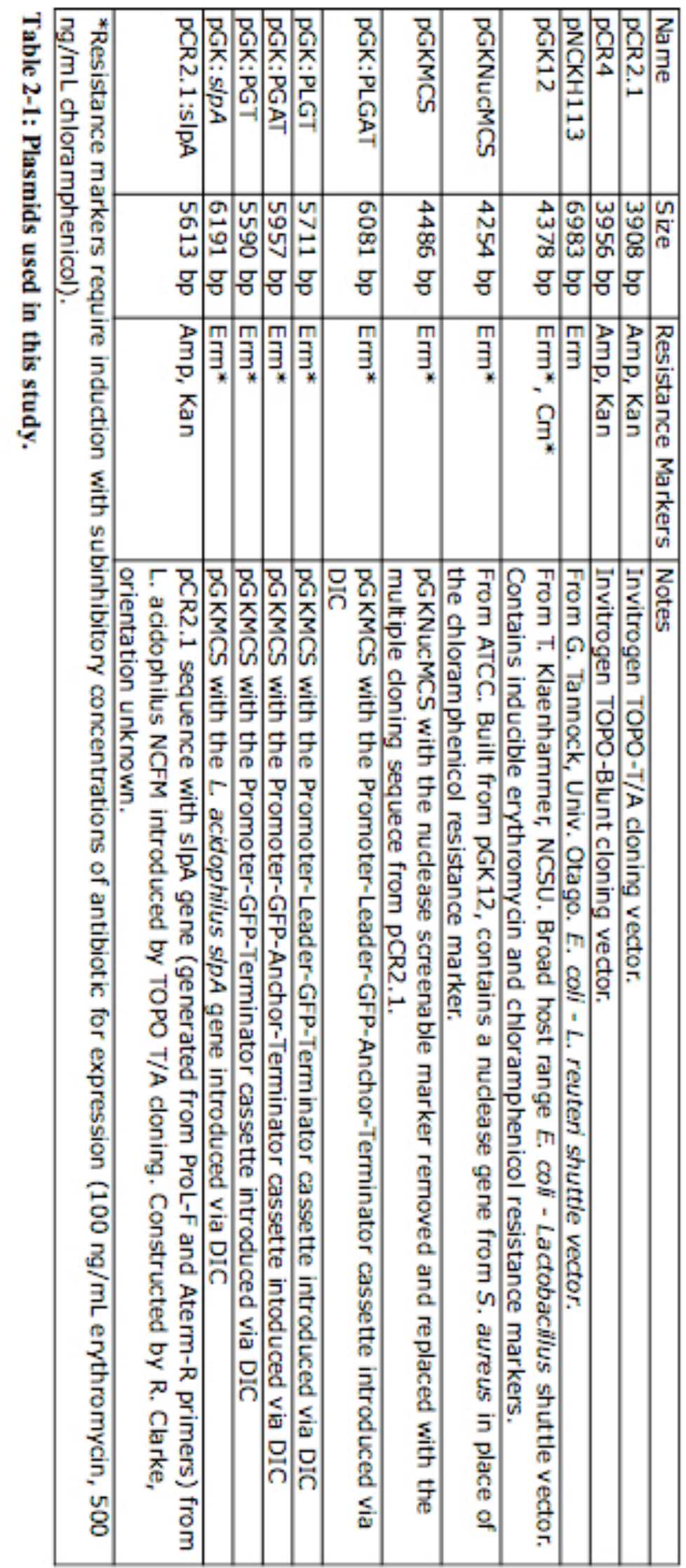




\subsubsection{Molecular techniques}

PCR was performed using either Taq DNA polymerase (Promega) or Phusion DNA polymerase (Finnzymes). The primers used in this study are listed in Table 2-1. For Taq amplifications, a $25 \mathrm{uL}$ reaction volume was used with $2.5 \mathrm{mM} \mathrm{MgCl}_{2}, 0.4 \mathrm{uM}$ of each primer, $0.8 \mathrm{mM}$ dNTPs, 1 unit of enzyme and $1 \mathrm{uL}$ of template DNA. Typical thermal cycling parameters for Taq DNA polymerase were as follows: Pre-denaturation at $96^{\circ} \mathrm{C}$ for 2 minutes; 30 cycles of $96^{\circ} \mathrm{C}$ for 30 seconds, $55^{\circ}$ for 30 seconds and $72^{\circ} \mathrm{C}$ for 1 minute/kb; a final elongation of 5 minutes at $72^{\circ} \mathrm{C}$ finished the reaction. Phusion amplifications were performed in a $20 \mathrm{uL}$ reaction volume containing $0.5 \mathrm{uM}$ of each primer, $0.8 \mathrm{mM}$ dNTPs, 1 unit of enzyme and $1 \mathrm{uL}$ of template DNA. Thermal cycling parameters for Phusion DNA polymerase were according tot the manufacturer's instructions: Pre-denaturation at $98^{\circ}$ for 1 minute; 30 cycles of $98^{\circ} \mathrm{C}$ for 30 seconds, $55^{\circ} \mathrm{C}$ for 30 seconds and $72^{\circ} \mathrm{C}$ for $30 \mathrm{~s} / \mathrm{kb}$; a final 2 minute extension at $72^{\circ} \mathrm{C}$ concluded the reaction. When colony PCR was used to screen for recombinant clones, a Taq reaction was set up as described previously except that the template consisted of raw cells from a potential clone.

Restriction enzyme digestions were performed using approximately 1 ug of DNA according to the reaction conditions suggested by NEB. If the digest was diagnostic, a 10 $\mathrm{uL}$ reaction volume was used, if the product was to used for downstream application such as ligation, the digest was performed in 20-30 uL reaction volumes using the appropriate 10X reaction buffer (NEB). Double digestions were performed in the buffer recommended by NEB. Sequential digestions were performed in two separate digestion reactions, each containing the optimal buffering conditions for the enzyme to be used. 
After the first digest was performed the intermediate DNA product was gel or column purified (Gel Spin, Zymo research) and the eluted product was digested in a second reaction with the suggested buffering conditions for the second enzyme.

Ligations were performed in $10 \mathrm{uL}$ reaction volumes. For classical cloning of cassette DNA into plasmids, a 4:1 insert:vector molar ratio was used with approximately 250 ng of linearized plasmid DNA. For cassette construction, a 1:1 ratio between components was used. The reactions were ligated at room temperature or $16^{\circ} \mathrm{C}$ for 30 minutes to 2 hours. After incubation plasmid ligations were transformed into E. coli. 


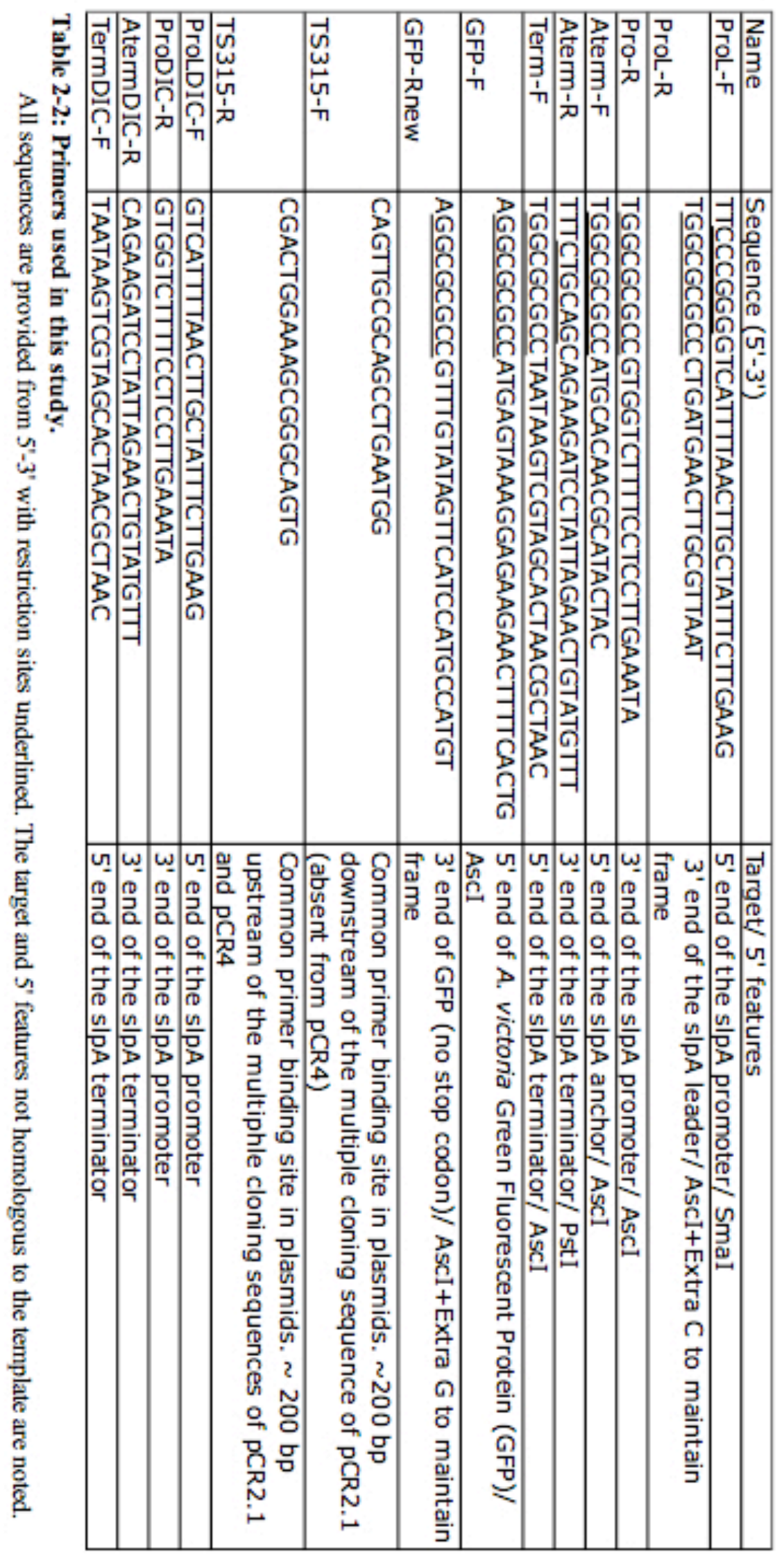




\subsubsection{Transformation of plasmid DNA into E. coli}

After ligation, $5 \mathrm{uL}$ or $1 \mathrm{uL}$ of the ligation reaction was transformed into chemically or electrocompetent $E$. coli, respectively, according to manufacturers instructions. When transforming pGK12-derived plasmids, a subinhibitory concentration of erythromycin $(100 \mathrm{ng} / \mathrm{mL})$ was added to the recovery medium (SOC) to induce expression of the ErmC promoter and the recovery time was extended to two hours. After recovery and/or induction the cells were plated onto selective media.

\subsubsection{Transformation of plasmid DNA into Lactobacillus}

Two different transformation methods were utilized to transform Lactobacillus acidophilus and Lactobacillus reuteri. For L. acidophilus a modified method from Kim et al. (Kim et al., 2005) was used. An overnight culture of L. acidophilus, grown in MRS, was used to inoculate $5 \mathrm{~mL}$ of MRS $+1 \%$ glycine (1:50 initial inoculum), the culture was grown until $\mathrm{OD}_{600}=0.3$ and the cells were harvested by centrifugation at $4000 \mathrm{Xg}$ for 10 minutes at $4^{\circ} \mathrm{C}$. The cells were washed $2 \mathrm{X}$ with ice-cold wash buffer $\left(5 \mathrm{mM} \mathrm{NaPO}_{4}, 1\right.$ $\mathrm{mM} \mathrm{MgCl} 2, \mathrm{pH} 7.4)$ and resuspended in $1 / 10$ volume electroporation buffer (1 M sucrose, $3 \mathrm{mM} \mathrm{MgCl}_{2}, \mathrm{pH}$ 7.4). After resuspension, $80 \mu \mathrm{L}$ of competent cells were added to an electroporation cuvette with an inner-electrode gap of $0.2 \mathrm{~cm}$ (Bio-Rad). Next, $5 \mu \mathrm{L}$ of plasmid DNA $(\sim 1 \mathrm{ug})$ was added to the cells and the mixture was incubated on ice for 10 minutes. A single pulse of $1.5 \mathrm{kV}$ and $480 \Omega$ was applied for an approximate pulse length of $16 \mathrm{~ms}$. Immediately following the pulse, the cells were recovered in $1.0 \mathrm{~mL}$ prewarmed MRS for 20 minutes at $37^{\circ} \mathrm{C}$. If the plasmid required induction, (pGK12-derived 
plasmids) then erythromycin was added to $100 \mathrm{ng} / \mathrm{mL}$ and incubated a further 3 hours at $37^{\circ} \mathrm{C}$. After induction, $250 \mu \mathrm{L}$ of the recovered cells were inoculated into $5 \mathrm{~mL}$ MRS with $5 \mu \mathrm{g} / \mathrm{mL}$ erythromycin and incubated either anaerobically or in candle jar overnight for the enrichment of transformants. After enrichment, $50 \mu \mathrm{L}$ was spread onto MRS with $5 \mu \mathrm{g} / \mathrm{mL}$ erythromycin plates and incubated for 48 hours in a candle jar until colonies were visible.

For Lactobacillus reuteri a modified transformation procedure from Ahrne et al. was utilized (Ahrne et al., 1992). MRS was inoculated with a 1:100 initial inoculum from a fresh overnight MRS broth culture. The cells were grown to $\mathrm{OD}_{600}=0.6$ and harvested by centrifugation as described above. The cells were washed $2 \mathrm{X}$ with ice-cold water and $1 \mathrm{X}$ with $1 / 10$ volume of EHR (1mM HEPES, $0.5 \mathrm{M}$ raffinose, $\mathrm{pH} 7.0$ ) buffer. The cells were then resuspended in $1 / 100$ volume of EHR buffer and $100 \mu \mathrm{L}$ aliquots were made in $1.5 \mathrm{~mL}$ microcentrifuge tubes. Approximately $5 \mu \mathrm{L}(\sim 1 \mu \mathrm{g})$ of plasmid DNA was added to $100 \mu \mathrm{L}$ aliquots and incubated on ice for 10 minutes. The remainder of the electroporation and recovery/selection procedure was the same as for L. acidophilus.

\subsubsection{Genomic DNA extraction from Lactobacillus}

Lactobacillus DNA extraction was performed as previously described (Dupont et al., 1995). Briefly, strains were grown in MRS broth $(10 \mathrm{~mL})$ for 48 hours and resuspended in $1.2 \mathrm{~mL}$ of TES (100 mM Tris-HCl pH 7.9, $20 \mathrm{mM}$ EDTA, $20 \%$ sucrose) with lysozyme $(25 \mu \mathrm{M} / \mathrm{ml})$ and mutanolysin $(5 \mathrm{U} / \mathrm{ml})$ and further incubated for $30 \mathrm{~min}$ at $37^{\circ} \mathrm{C}$. Then, $80 \mu \mathrm{L}$ of $10 \%$ SDS with proteinase $\mathrm{K}(400 \mu \mathrm{g} / \mathrm{ml})$ was added and the 
mixture was incubated for $30 \mathrm{~min}$ at $60^{\circ} \mathrm{C}$. DNA was extracted by the phenol:chloroform method and ethanol precipitated. DNA was resuspended in $50 \mu \mathrm{L}$ TE $(10 \mathrm{mM}$ Tris- $\mathrm{HCl}$, 1 mM EDTA) to be used in PCR.

\subsubsection{Construction of S-layer GFP fusion cassettes}

All components necessary to build the S-layer GFP reporter cassettes were amplified from extracted Lactobacillus acidophilus NCFM genomic DNA or plasmid pMB293 (source of GFP) using the high fidelity enzyme, Phusion DNA polymerase (Finnzymes). Each cassette was designated according to the first letter of the components present in the given construct. For example, PLGAT contained the slp $A$ promoter, leader, anchor and terminator as well as GFP as a reporter. For simplicity only the construction of one cassette (PLGAT) will be provided here in detail, all other cassettes were built analogously. GFP 5' Seq contains only the 5' $\sim 100$ nucleotides of GFP (and the 5' AscI site) and was necessary to build the constructs by overlap extension PCR due to the nonhomologous clamps required for efficient restriction enzyme cleavage. After amplification the Promoter-Leader, GFP, GFP 5'Seq and Anchor-Terminator amplicons were digested with AscI (NEB) and gel purified (Gel Spin, Zymo Research) following manufacturers instructions. Promoter-Leader-GFP and GFP5'Seq-Anchor-Terminator were ligated as described above, the partial cassette was then amplified using ProL-F/ GFP-Rnew or GFP5'Seq/Aterm-R primer pairs respectively. The amplified products of the expected sizes were gel purified and diluted 1:50. Next, $1 \mu \mathrm{L}$ of each diluted product was mixed together in a $20 \mu \mathrm{L}$ Phusion PCR reaction containing the primers ProL- 
F/Aterm-R. The amplicon of the expected size was gel purified and stored at $-20^{\circ} \mathrm{C}$ until use.

2.2.7 Construction of the pGKMCS shuttle plasmid

pGKMCS was built according to Figure 2-2 Briefly, the multiple cloning sequence (MCS) of pCR2.1 (Invitrogen) was amplified using TS315-F and TS315-R primers, which bind approximately 200 nucleotides flanking the MCS using Phusion DNA polymerase. The amplicon was gel purified and digested with NsiI. The plasmid pGKNucMCS was digested with XhoI and end filled using $T_{4}$ DNA polymerase (NEB). The end-filling reaction contained 3 units of $\mathrm{T}_{4}$ DNA polymerase, $0.1 \mathrm{mM}$ dNTPS, $\sim 2$ $\mu \mathrm{g}$ of freshly digested and heat inactivated plasmid and $1 \mathrm{X}$ NEB buffer 3 in a $25 \mu \mathrm{L}$ reaction volume. The reaction was incubated at $12^{\circ} \mathrm{C}$ for 15 minutes then heat inactivated at $65^{\circ} \mathrm{C}$ for 20 minutes. The plasmid was then digested with NsiI and gel purified. A blunt/sticky end ligation was performed to clone the insert into the linearized vector. This ligation was performed at room temperature overnight. The ligation product was transformed into chemically competent TOP 10 , induced for 2 hours and plated onto selective media (LBE250). Successful recombinant clones were confirmed by plasmid extraction and diagnostic digests using enzymes unique to the newly introduced MCS. 

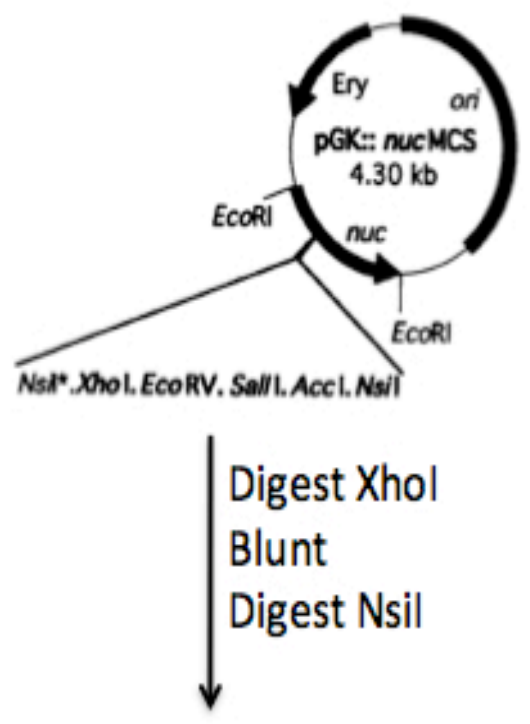

Ligate TS315-R $\rightarrow$ Nsil Into blunted Xhol and Nsil

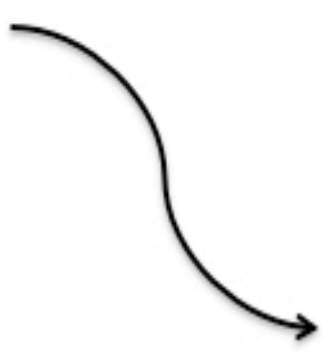

Figure 2-2: Construction of shuttle plasmid pGKMCS.

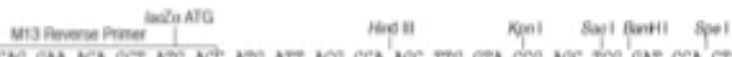

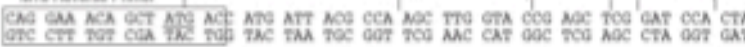

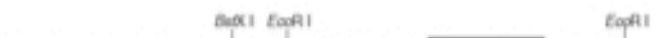

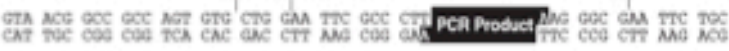

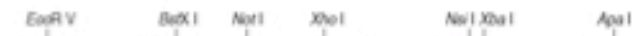

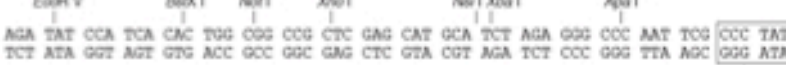

unctorame iom prese

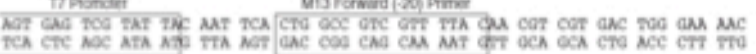
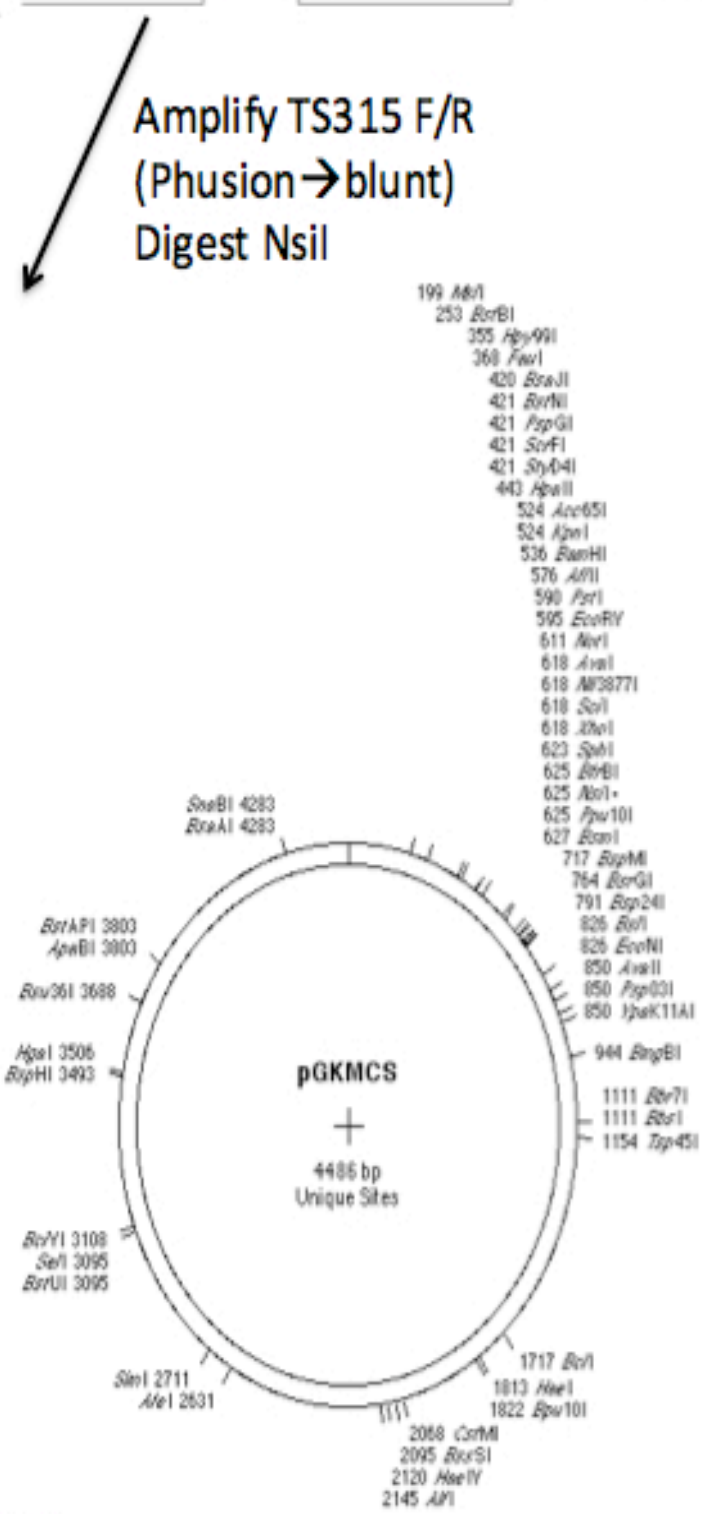

253 Rere

15s

CO BSWNI

ol

स1 $\sin 4$

ind

524 ine6s

30 Ruot

S9t $/$ gat

ITS Euthy

618 Anai

pGKMCS was built by incorporating the multiple cloning site from pCR2.1 into the pGKMCS shuttle plasmid (see text for details). 
2.2.8 Classical cloning of S-layer GFP cassettes into shuttle plasmids

When the target cloning plasmid was pNCKH113 both the plasmid and insert were digested either simultaneously or sequentially with XmaI and PstI. When cloning into pGKMCS the cassette was first digested with $\mathrm{SmaI}$ at $25^{\circ} \mathrm{C}$ for 1 hour, then PstI was added and digested at $37^{\circ} \mathrm{C}$ for a further 1 hour. The vectors and inserts were ligated and transformed as described above.

\subsubsection{Digestion independent cloning of S-layer GFP cassettes into pGKMCS}

Digestion independent cloning (DIC) was performed essentially as described in Figure 2-3 except the cloning reaction only involved two steps instead of three (the annealing step was omitted). The donor vector, pGK:slpA, was constructed by first amplifying the full length $s l p A$ gene using Taq DNA polymerase from $L$. acidophilus NCFM genomic DNA using ProL-F and Aterm-R primers. The slpA gene was then cloned into pCR2.1 by the manufacturers instructions (R. Clarke). Next, the $\operatorname{slp} A$ gene along with $\sim 200 \mathrm{bp}$ of flanking sequence was amplified from pCR2.1:slp $A$ with the TS315 F/R primer pair. The amplicon was then digested with EcoRI and ligated into the EcoRI digested pGKMCS plasmid and transformed into chemically competent TOP10 cells. The DIC vector was amplified with Phusion DNA polymerase using TermDIC-F and ProDIC-R primers. The cassettes were amplified using ProLDIC-F and AtermDIC-R primers and the amplicons were column purified. The vector amplicon was then digested with DpnI to remove residual pGK:slpA and heat inactivated for 20 minutes at $65^{\circ} \mathrm{C}$. Approximately $500 \mathrm{ng}(2 \mu \mathrm{L})$ of vector amplicon was mixed with the appropriate amount 
of insert amplicon to achieve a 3:1 molar ratio of insert to vector. The DIC reaction contained $4 \mu \mathrm{L}$ of the supplied Phusion high fidelity (HF) buffer, $1.6 \mu \mathrm{L}$ of $10 \mathrm{mM}$ dNTPs, $0.2 \mathrm{uL}$ Phusion DNA polymerase and PCR grade water up to $20 \mu \mathrm{L}$. Thermocycling parameters consisted of pre-denaturation at $98^{\circ} \mathrm{C}$ for 60 seconds, then 5 cycles of $98^{\circ} \mathrm{C}$ for 15 seconds and $72^{\circ} \mathrm{C}$ for 120 seconds, a single final extension of 5 minutes at $72^{\circ} \mathrm{C}$ was used to fill in any remaining gaps and finish the reaction. Finally, 5 uL was transformed into chemically competent NEB Turbo cells. Possible recombinant clones were screened by colony PCR and fluorescence microscopy. 

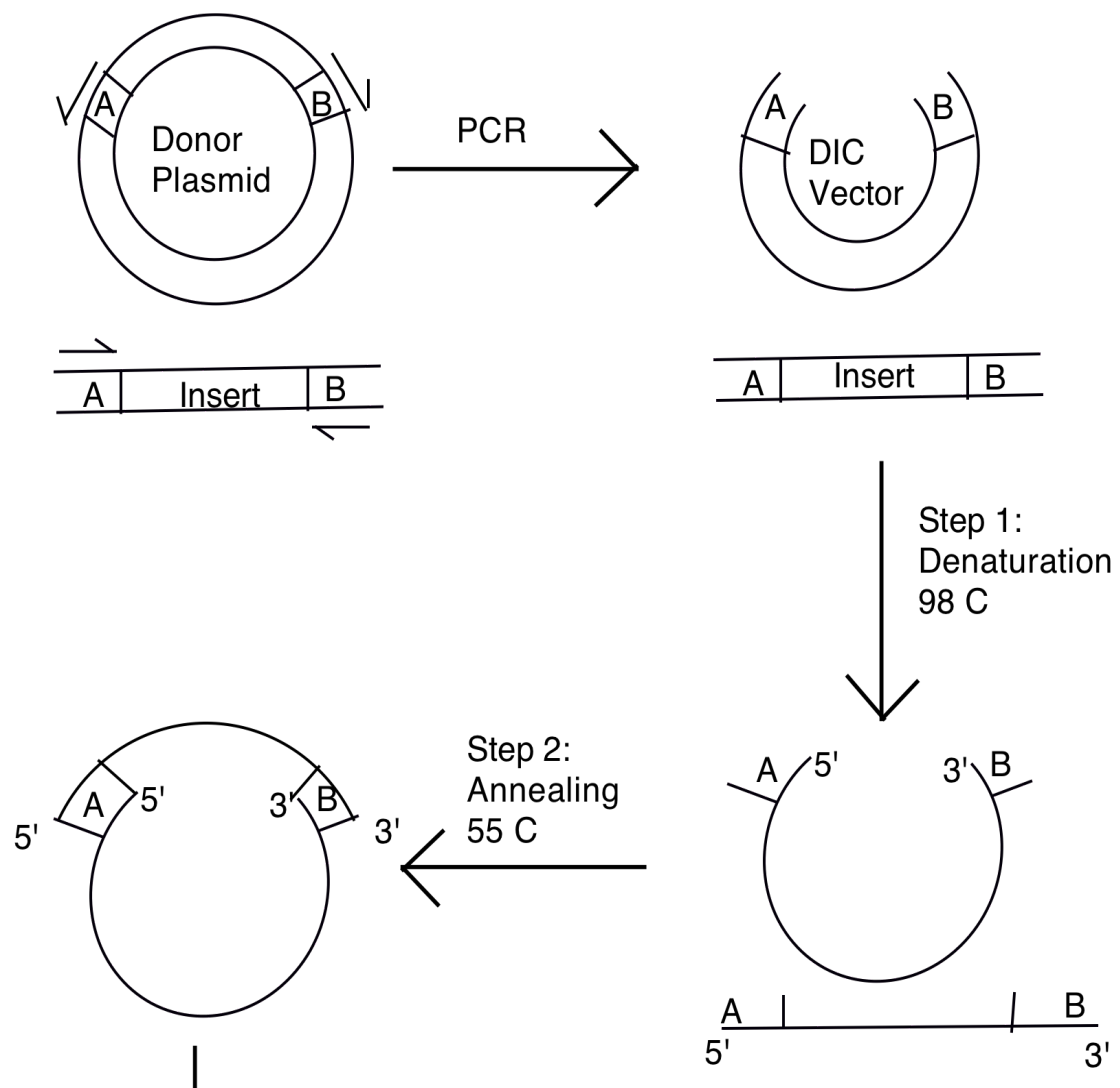

Step 3:

Extension

$72 \mathrm{C}$

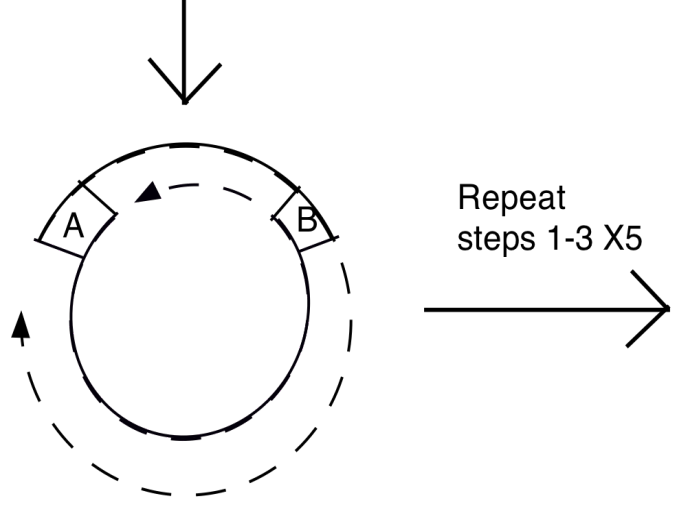

Figure 2-4: Digestion Independent Cloning (DIC)

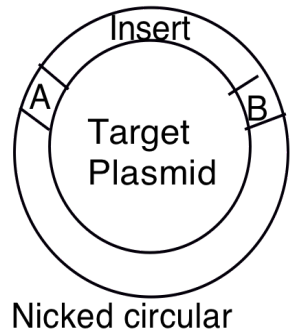

recombinant plasmid suitable for transformation.

Vector preparation: A donor vector containing homology with the $5^{\prime}$ and $3^{\prime}$ ends of the target insert is used as a template for amplification of the DIC vector.

DIC: A three-step DIC reaction is outlined. Denaturation creates single stranded DNA (for simplicity only one strand is shown). The two molecules interact via base pairing of homologous sequences during the annealing step. During the extension step the $3^{\prime}$ ends of each molecule is elongated to fill the single stranded gaps, forming double stranded DNA. 5 cycles allows for enough target plasmid to recover recombinant clones after transformation. 


\subsubsection{Detection of GFP expression by western blot}

Whole cell lysates were prepared from NEB Turbo E. coli cells harboring pGK:PLGAT, pGK:PLGT, pGK:PGAT, pGK:PGT and pGK:slpA. First, 3 mLs of cells were harvested after growth to $\mathrm{OD}_{600}=0.5$, resuspended in PBS $+0.1 \%$ Triton X-100 and lysed by sonication $2 \mathrm{X}$ for 10 s at power setting 5 ( 14 RMS). Next, $22.5 \mu \mathrm{L}$ of each whole cell lysate was added to $7.5 \mu \mathrm{L}$ of $4 \mathrm{X}$ SDS sample buffer $(40 \%$ glycerol, $240 \mathrm{mM}$ Tris/ $\mathrm{HCl} \mathrm{pH} 6.8,8 \%$ sodium dodecyl sulfate, $0.04 \%$ bromophenol blue and $5 \% \beta$ mercaptoethanol). The samples were then boiled at $99^{\circ} \mathrm{C}$ for 5 minutes and placed on ice or stored at $-20^{\circ} \mathrm{C}$ until analysis.

For western blot analysis, $20 \mu \mathrm{L}$ of each sample was electrophoresed for $60-80$ minutes @ 120V until the dye reached the bottom of the gel (12\% polyacrilamide). The proteins were transferred to a nitrocellulose membrane at $350 \mathrm{~mA}$ for 1 hour or $35 \mathrm{~mA}$ overnight. Successful transfer was confirmed by ponceau staining and the molecular weight standards were marked. The membrane was then blocked in $3 \%$ non-fat dry milk in PBS-Tween $(0.1 \%) \mathrm{pH} 7.4$ for 3 hours (at room temperature) to overnight $\left(\right.$ at $4^{\circ} \mathrm{C}$ ) with gentle rotation. Three washes with $15 \mathrm{~mL}$ PBS-Tween for 10 minutes each were performed. This wash step was repeated after the primary and secondary antibody incubations. The primary antibody was polyclonal chicken anti-GFP. The secondary antibody was horseradish peroxidase (HRP) conjugated goat anti-chicken. Each antibody was diluted 1:2000 in 10 mL PBS-Tween, added to the blot and incubated at room temperature for 1 hour with shaking. Both the primary and secondary antibodies were 
kindly provided by John Goers of the Biochemistry Department Cal Poly, SLO. HRP was detected by ECL + (Amersham Biosciences) and visualized by chemiluminescent detection.

\subsubsection{Plasmid sequencing}

Sequencing of cloned S-layer GFP inserts from the plasmids pGK:PLGAT, pGK:PLGT, and pGK:PGT using the primers ProL-F and M13-R was performed by Seqwright DNA Technology Services. The received sequence chromatograms were visualized using 4Peaks software (A. Griekspoor and Tom Groothuis, mekentosj.com). The sequence was manually edited to remove the ends with poor sequence data. For pGK:PGT the forward and reverse sequencing reads were of sufficient length to allow for manual contig assembly. This contig was then aligned to the expected sequence. When contigs could not be assembled (pGK:PLGAT and pGK:PLGT) the individual sequencing reads were aligned to the expected sequences. Any suspected mutations were confirmed by inspecting the chromatograms. 


\subsection{Results}

\subsubsection{Construction of GFP S-layer cassettes}

Successful construction of the four GFP cassettes containing the expression (promoter and terminator) and localization signals (leader and anchor) was initially performed with Taq DNA polymerase. Subsequently, all cassettes were re-built with Phusion DNA polymerase. Some non-target background amplification was seen in all full-length amplicons necessitating gel purification of the desired products according to size. To confirm that the junctions had been ligated correctly, all cassettes were digested with AscI (Figure 2-4). Each cassette contained all of the expected individual components from Table 2-3.

\begin{tabular}{|l|l|l|}
\hline S-layer GFP Cassettes & Size & Expected Localization \\
\hline Promoter-Leader-Gfp-Anchor-Terminator (PLGAT) & $1595 \mathrm{bp}$ & Exterior cell wall \\
\hline Promoter-Leader-GFP-Terminator (PLGT) & $1225 \mathrm{bp}$ & Extracellular \\
\hline Promoter-GFP-Anchor-Terminator (PGAT) & $1471 \mathrm{bp}$ & Cytoplasm \\
\hline Promoter-GFP-Terminator (PGT) & $1104 \mathrm{bp}$ & Cytoplasm \\
\hline & & \\
\hline Individual Components & Size & \\
\hline Promoter-Leader & $376 \mathrm{bp}$ & \\
\hline Promoter & $256 \mathrm{bp}$ & \\
\hline Anchor-Terminator & $504 \mathrm{bp}$ & \\
\hline Terminator & $135 \mathrm{bp}$ & \\
\hline Green Fluorescent Protein (GFP) & $732 \mathrm{bp}$ & \\
\hline
\end{tabular}

Table 2-3: S-layer GFP cassettes.

The expected sizes and cellular localization of each S-layer GFP reporter cassette are provided. The sizes of each component of the cassettes is also stated. 


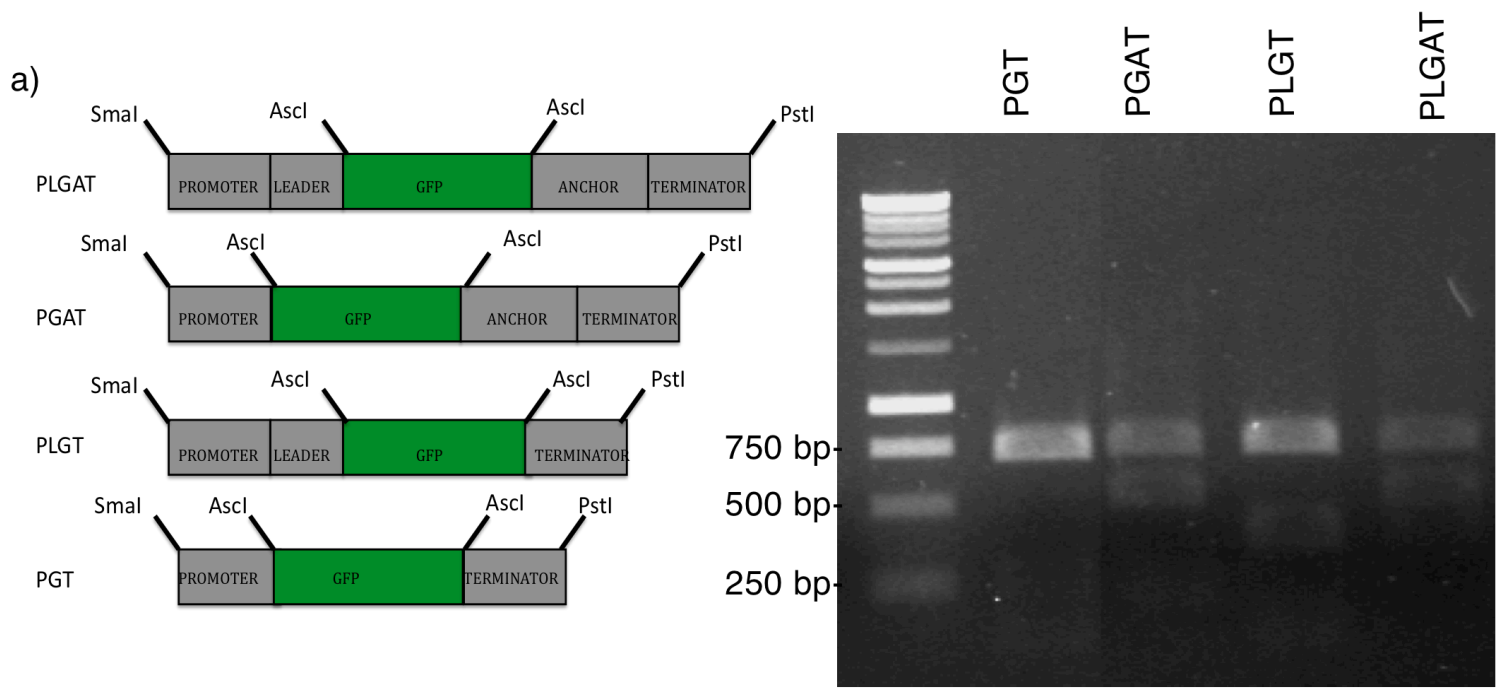

Flgure 2-4: Construction of the S-layer GFP cassettes.

a) Graphical representation of each S-layer cassette.

b) Each gel purified Phusion amplicon was digested with Ascl to confirm successful construction. The expected sizes for each compnent are provided in Table 2-3.

2.3.2 Transformation of shuttle vectors into E. coli, L. acidophilus and L. reuteri

Repeated initial attempts to transform pGK12 and pGKNucMCS into various strains of E. coli (TOP10, DH5 $\alpha$, T-1 and NEB Turbo) were unsuccessful unless a 1-2 hours induction period was included during the recovery stage (see Materials and Methods). Transformation of pGK12 and pCKNucMCS into L. acidophilus and $L$. reuteri after induction were successful resulting in antibiotic resistant colonies after 48 hours.

Transformation of pNCKH113 into E. coli TOP10 and into L. reuteri ATCC 23272 was assumed to be successful, as plasmid DNA of the appropriate size and digestion pattern for pNCKH113 was regularly recovered from E. coli and L. reuteri. However, repeated transformation attempts into L. acidophilus NCFM were 
unsuccessful. To determine if the inability to recover erythromycin resistant clones was due to incompatibility of this plasmid in L. acidophilus or if L. acidophilus was simply not amenable to transformation, pGK12 was transformed into L. acidophilus. Transformation of pGK12 into L. acidophilus resulted in antibiotic resistant clones after $\sim 48$ hours, suggesting a problem with replication, selection or maintenance of pNCKH113 in L. acidophilus. In order to achieve a sufficiently broad host range, the pGKNucMCS derived plasmid, pGKMCS was utilized for all cassette cloning. The pGKMCS shuttle plasmid contains the multiple cloning sequence from pCR2.1 as well as the origin and erythromycin resistance marker from pGK12 and was able to be transformed into all organisms tested. The transformation efficiencies in L. reuteri and $L$. acidophilus NCFM were very low $\left(<10^{2}\right.$ and $<10 \mathrm{cfu} / \mathrm{ug}$ respectively), in spite of multiple attempts to optimize the electroporation parameters.

\subsubsection{Cloning of S- layer GFP cassettes into pGKMCS and expression in E. coli}

The insertion of S-layer GFP cassettes into pGKMCS using classical cloning techniques was repeatedly unsuccessful. Likewise, sub-cloning of the cassettes into pCR2.1 or pCR 4 TOPO cloning vectors resulted only in the recovery of clones that did not contain the inserts of interest. However, sub-cloning of the wild type $\operatorname{slp} A$ gene (amplified from L. acidophilus genomic DNA using ProL-F and Aterm-R) into pCR2.1 and subsequent ligation into pGKMCS was successful.

To efficiently clone the GFP reporter cassettes into pGKMCS a digestion independent cloning (DIC) technique was developed (Figure 2-3). DIC allows for 
orientation specific cloning using sequence homology between the insert and vector. For DIC, insert and vector DNA containing homology at each end was mixed together in a PCR extension reaction without primers and taken through 5 cycles. A relatively high degree of success was found with this method, allowing the recovery of $>100$ potential clones for each cassette. Recovered clones were screened by PCR to identify those that were carrying the expected expression cassettes. After the clones were identified by PCR, they were screened for GFP expression by fluorescence microscopy. Clones that were GFP positive and PCR positive were identified for all cassettes except for PLGAT, which only yielded PCR positive clones (Figure 2-5).

PLGT positive clones were brightly fluorescent. This fluorescence however, was not uniform throughout the population. PGAT clones were comparatively faintly fluorescent with only a minority of cells within the population showing any fluorescence. PGT clones showed the most uniform fluorescence and were also bright, though slightly duller than PLGT. No fluorescence above background could be observed with any Slayer GFP cassette bearing plasmids when transformed into L. acidophilus (Figure 2-5).

To confirm the expression of GFP and determine the size of the proteins, clones bearing each insert were analyzed by western blotting. All clones except pGK:PLGAT yielded distinctive GFP fusion products with bands at approximately the expected sizes of $40.4 \mathrm{kD}, 31.1 \mathrm{kD}$ and $28.8 \mathrm{kD}$ for PGAT, PLGT and PGT respectively (Figure 2-6). Since the PLGT component contained the leader sequence, the molecular weight was dependent on whether or not this component is cleaved in E. coli (Figure 2-1). Since the $2.3 \mathrm{kD}$ difference in size cannot be resolved on this percentage acrylamide gel (12\%), it is impossible to definitively ascertain whether or not this element is properly processed 
from Figure 2-6. As a positive control for GFP, Saccharomyces cerevisiae expressing a Pep12-GFP fusion protein (52.8 kD) was used. A negative control E. coli strain containing a plasmid with the wild type $\operatorname{slp} A$ gene was also included for comparison. 


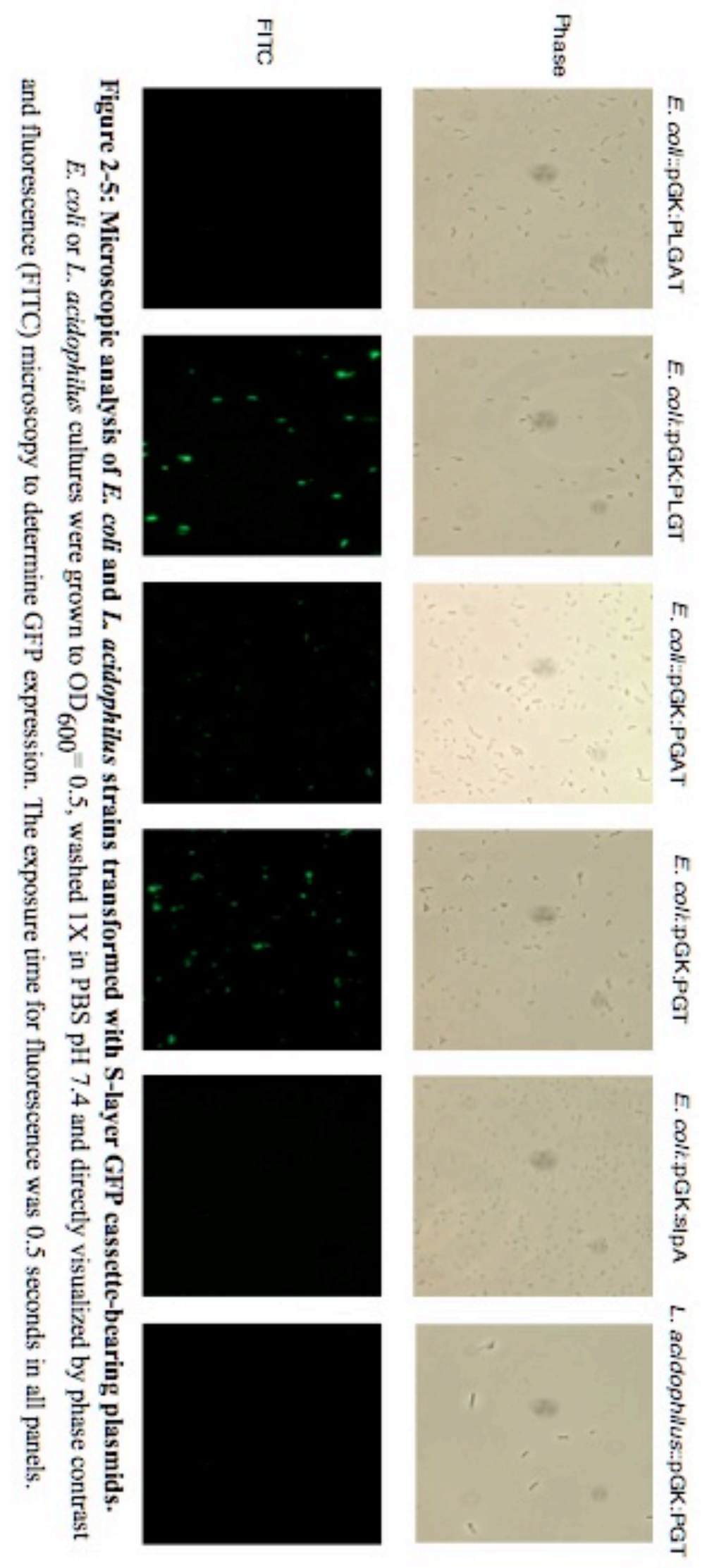




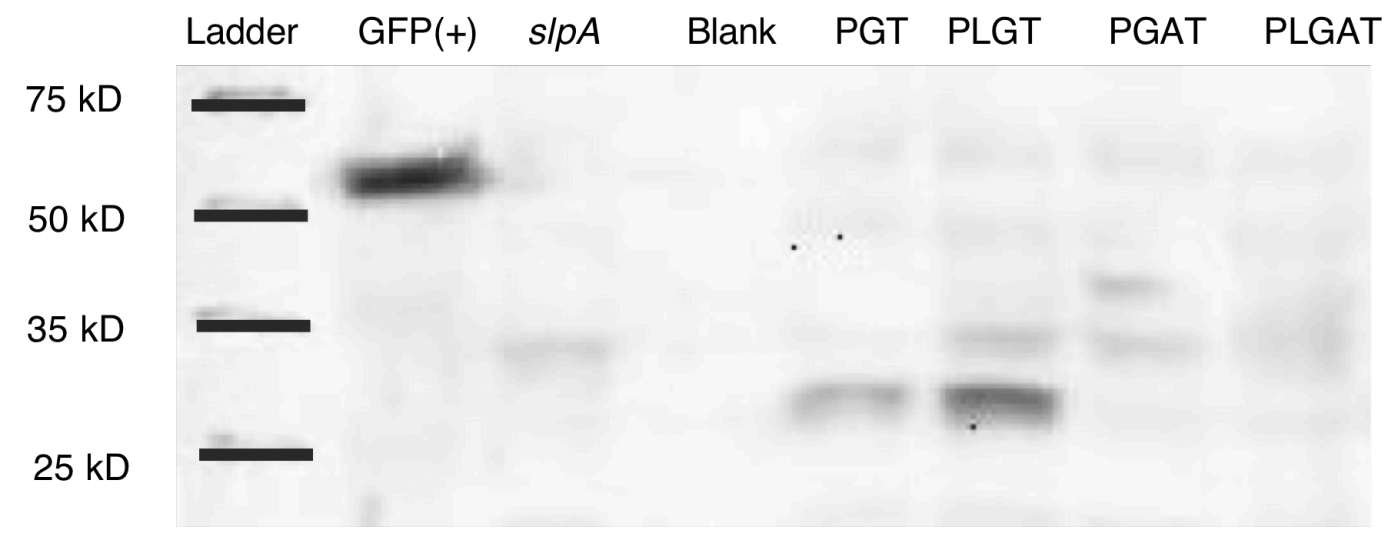

Figure 2-6: GFP expression in $\boldsymbol{E}$. coli containing S-layer GFP cassettes

GFP expression in E. coli strains bearing the plasmids pGK:PLGAT, pGK:PLGT, pGK:PGAT and pGK:PGT as determined by western blot the Pep12-GFP fusion acted as a GFP positive control and $\mathrm{pGK}$ :slpA was included as a negative control. The expected sizes of the expressed proteins from each cassette were PLGAT: 45.1 (pro-protein-P) or 42.8 (secreted form S); PGAT: 40.4 kD; PLGT: 31.1 (P), 28.8 (S); PGT: 27.1 kD; GFP (+) Pep12-GFP: 52.8 kD.

2.3.4 Sequencing of the plasmids pGKMCS:PLGAT, pGKMCS:PLGT and pGKMCS:PGT

Three plasmids, pGKMCS:PLGAT, pGKMCS:PLGT and pGKMCS:PGT were sequenced to elucidate reasons why PLGAT is not expressed in E. coli or Lactobacillus and why no GFP expression can be visualized in Lactobacillus. The sequencing data is summarized in Figure 2-4. Results for the PLGAT cassette showed a frameshift deletion of two nucleotides at places CDS\# 137, 138 (CDS = coding sequence), which resulted in an early stop codon at CDS\# 175-177. The PLGT cassette acquired a single nucleotide insertion at CDS\# 105 directly resulting in a stop codon. Due to the length of the PLGAT and PLGT cassettes the forward and reverse sequence reads were not long enough to build contigs resulting in gaps in the sequence alignment. The gaps for the PLGAT and 
PLGT cassettes were from CDS\# 430-745 and CDS\# 463-484 respectively. For the available sequence, the PGT insert appeared to be as expected, however, poor sequence data was obtained for the 5' end of each insert, including the sigma factor binding sites. The percent coverage for each cassette was calculated by number of quality base reads by the expected size for each cassette and multiplied by 100 to express as a percentage. The percent coverage for the three inserts was 77.77, 95.43 and 95.56 for PLGAT, PLGT and PGT respectively. 


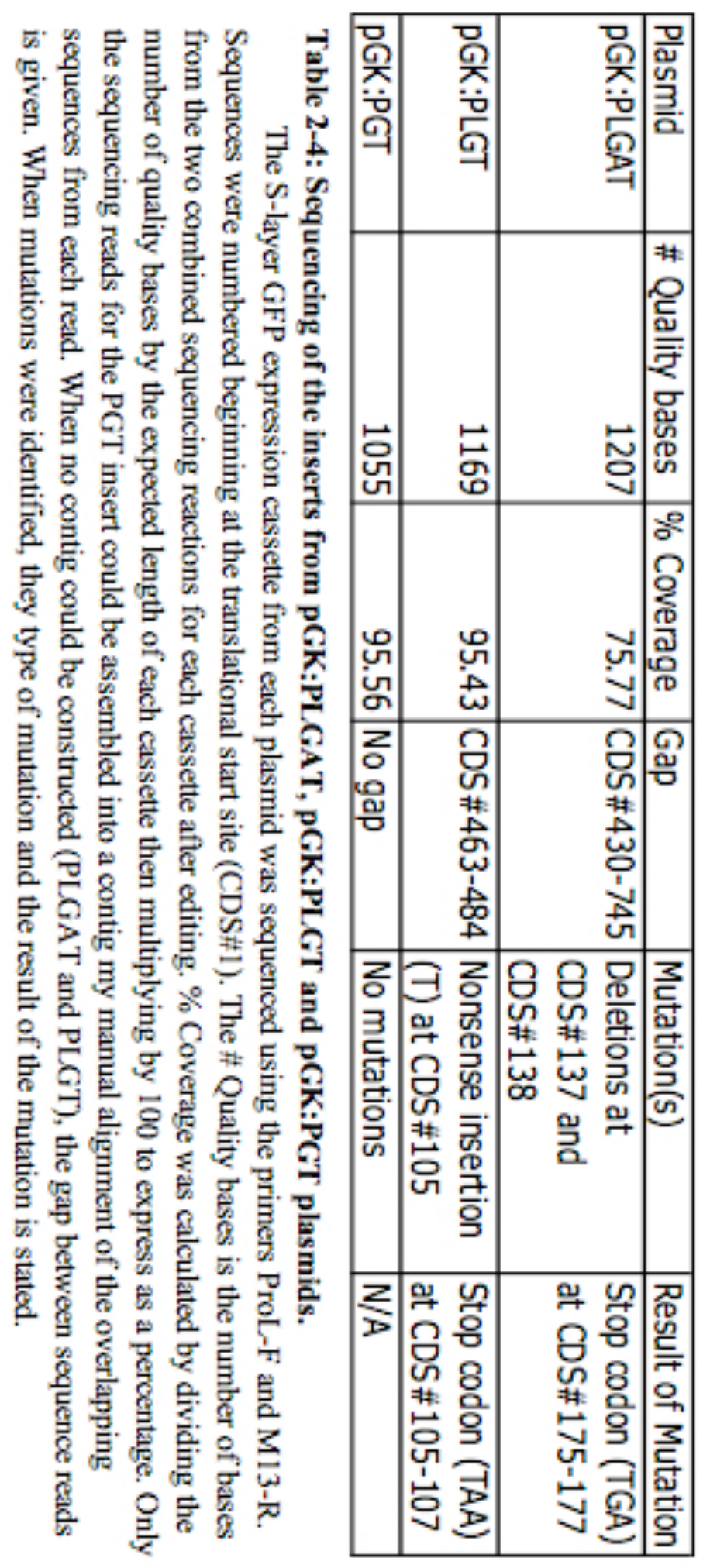




\subsection{Discussion}

The construction of GFP reporter constructs to assess the localization and expression signals of $\operatorname{slp} A$ expression was successful as determined by diagnostic digest patterns. While the sequence of the complete cassette could not be assessed by restriction pattern alone, the banding pattern suggests that each insert contains the expected component and that the AscI restriction site at the junction of each element is conserved. All amplifications were performed with high fidelity (manufacturer reported error rate =

$4.4 \times 10^{-7}$ ) Phusion DNA polymerase making the likelihood of mutations being generated in these cassettes due to PCR synthesis highly unlikely.

The usefulness of the selected the E. coli-Lactobacillus shuttle vectors was determined by the criteria that a suitable vector must be easily transformed into $E$. coli, possess a sufficiently broad host-range allowing introduction into various Lactobacillus species and contain an adequate multiple cloning site for insertion of heterologous DNA. None of the vectors initially obtained satisfied all of the above requirements. The pNCKH113 plasmid showed narrow host specificity, as it would not transform $L$. acidophilus NCFM. It was subsequently learned that the erythromycin resistance gene harbored by this plasmid (ermGT) is not expressed in L. acidophilus (Nicholas Heng, Personal communication). Therefore, it is possible that by replacing this antibiotic resistance determinate with another that is expressed in L. acidophilus (i.e. the erythromycin or chloramphenicol resistance genes from pGK12) a suitably broad host range plasmid could be produced. The pGK12 plasmid and its derivative pGKNucMCS, 
which has the chloramphenicol resistance gene replaced with a nuclease gene from $S$. aureus as a screenable marker, could not initially be transformed into E. coli. Both chemically competent and electrocompetent cell transformation techniques were used to initially introduce these plasmids into a variety of $E$. coli strains, all without success. Though not widely reported in published material describing the use of these plasmids, a literature search of the origin of the erythromycin and chloramphenicol resistance genes resulted in the realization that both antibiotic resistance markers are under control of inducible promoters. In our hands, induction during the recovery period with subinhibitory concentrations of erythromycin $(100 \mathrm{ng} / \mathrm{mL})$ or chloramphenicol (500 $\mathrm{ng} / \mathrm{mL}$ ) is an absolute requirement for the recovery of antibiotic resistant transformants.

The erythromycin resistance gene, ErmC, from pGK12 was originally encoded by a 3728 bp plasmid, pE194, initially isolated from Staphylococcus aureus (Hourinouchi \& Weisblum, 1982). The ErmC mRNA contains an extensive untranslated region, comprising two Shine Dalgarno sequences (SD1 and SD2), that is responsible for regulating expression of the protein. The remainder of the mRNA contains two open reading frames, one short reading frame constituting the leader and a long reading frame encoding the ErmC protein. The mechanism of ErmC inducible expression has since been extensively studied and is regulated by translational attenuation (Shivakumar et al., 1980; Gryczan et al., 1980; Dubnau, 1985; Hue \& Bechhofer, 1991; Mayford \& Weisblum 1989). When erythromycin is not present, only SD1 is accessible for ribosome binding and only the 19 amino acid leader peptide is synthesized. When erythromycin is present in low concentration, the erythromycin bound ribosome will stall at a specific place along the ErmC mRNA while translating the leader peptide, causing a conformational shift in 
the secondary structure of the mRNA and exposing the second Shine Dalgarno sequence, $\mathrm{SD} 2$. Once this sequence is accessible, the ribosome will bind to SD2 allowing for efficient translation of the ErmC gene product. After translation, the ErmC protein confers resistance by methylating the $23 \mathrm{~S}$ ribosomal subunit resulting in the inability of erythromycin to efficiently bind the ribosome and inhibit translation.

Once it was determined that pGK12 and pGKNucMCS could be readily transformed into all organisms tested by the inclusion of an induction step, a suitable shuttle vector with a robust multiple cloning sequence was built by incorporating the multiple cloning sequence from the common, commercially available cloning plasmid pCR2.1. The resulting plasmid pGKMCS contains all of the necessary requirements for the plasmid-based study of genetic components in Lactobacillus.

Classical cloning techniques utilizing digestion of GFP reporter cassette amplicons and the pGKMCS vector with the appropriate restriction enzymes and subsequent ligation with $\mathrm{T}_{4}$ DNA ligase were repeatedly unsuccessful. The sub-cloning of these amplicons into high efficiency topoisomerase-mediated (TOPO) cloning systems was also unsuccessful. Since the TOPO vectors utilized (pCR2.1 and pCR4) are high copy number vectors it is possible that sub-cloning was unsuccessful due to toxicity associated with the cassettes that was amplified by the copy number of the plasmid. Although, pGKMCS is a much lower copy number plasmid ( $\sim 60$ copies per cell in E. coli - Kok et al., 1984), it was possible that the inability to recover recombinant clones was due to the relative inefficiency of classical cloning using $\mathrm{T}_{4} \mathrm{DNA}$ ligase. To increase cloning efficiency, a novel method of digestion independent cloning (DIC) was developed. 
DIC utilizes homology at the 5' and 3' ends between a cloning vector and the desired insert as the driving force for cloning. By using pGK:slp $A$ as a template to amplify the pGK backbone along with the promoter and terminator components, we were able to generate a cloning vector with about 200 nucleotides of homology with the $5^{\prime}$ and 3' ends of each insert. Employing this cloning technique, all cassettes could be introduced into $E$. coli and variable degrees of fluorescence were observed. Perhaps most interesting were the observations that while PLGT and PGT were very bright PGAT fluorescence was comparatively dim and non-uniform within the population and PLGAT showed no fluorescence. This led to the hypothesis that there is toxicity associated with the anchor, resulting in lower expression/fluorescence from the introduced cassettes. Indeed, it was recently reported that the C-terminal region anchor region of the $\operatorname{slp} A$ gene has murein hydrolase activity, for which the $E$. coli peptidoglycan layer is a substrate (Acosta et al., 2008). The authors compared the C-terminal region of the $\operatorname{slp} A$ gene with two other known murein hydrlolases and found a nearly universally conserved sequence of 21 amino acids that spans the Sac1 and Sac2 domains (Acosta et al., 2008; Figure 21c). This discovery may also explain the difference in observed fluorescence between PLGAT and PGAT, as the peptidoglycan layer in E. coli is located in the periplasmic space. A leader driving secretion would be necessary for the anchor containing gene fusion product to efficiently infiltrate the periplasmic space. Accumulation of murein hydrolase activity in the E. coli periplasm could result in the inability to grow and divide, as well as possibly compromising the integrity of the cell. We therefore reasoned that toxicity is associated with this anchor region, especially when combined with the leader sequence under control of the high level constitutive expression of the $\operatorname{slp} A$ promoter. In 
the case of the PLGAT cassette, this anchor-mediated toxicity could result only in the recovery of clones with mutated inserts, due to selection against intact constructs. If this line of reasoning were accurate, the anchor-less cassettes would not have the same high degree of toxicity and would therefore not be under the same selective pressure as those containing an anchor. To confirm that the lack of fluorescence seen in PLGAT clones was in fact due to mutation, three plasmids, pGK:PLGAT, pGK:PLGT and pGK:PGT, were sequenced.

As the anchor-mediated toxicity hypothesis suggested, the plasmid containing the PLGAT insert included a deletion of two nucletides leading to a premature stop codon just within the coding region for GFP, thus abrogating expression.

Surprisingly, the sequence for the PLGT cassette also acquired a frameshift mutation leading to an early stop codon before the GFP open reading frame. There are two possible explanations for the PLGT sequence containing a frameshift mutation and yet still producing highly fluorescent cells. First, a frameshift read-through event occurred, allowing leaky expression of the leader-GFP fusion protein. Frameshift readthrough has been well documented in E. coli (Atkins et al. 1972). This phenomenon can occur in $E$. coli when the ribosome stumbles during translation and skips back into the proper reading frame before a stop codon is encountered. This is generally a rare event, resulting in greatly reduced expression of the mutated gene (Atkins et al. 1972), but may be amplified in this case due to increased stumbling by erythromycin bound ribosomes. Second, translation could have begun at the GFP start codon in the absence of classical translation initiation signals. The region upstream of the GFP start codon was analyzed to determine if any sequences responsible for recruiting ribosomes were present. Although 
there was no evidence to suggest a ribosome binding site, this possibility cannot be excluded. Finally, the limited expression of GFP by either of these scenarios may be enhanced by the very strong $\operatorname{slp} A$ promoter and the mRNA stabilizing effect of the $\operatorname{slp} A$ 5' UTR.

According to western blot, GFP products from pGK:PLGT and pGK:PGT migrate at the same rate during SDS-PAGE. This suggests that the product from pGK:PLGT is either not expressing the leader sequence (compatible with explanation two) or, that the leader region is being efficiently processed and the $1.8 \mathrm{kD}$ difference between PGT and the secreted form of PLGT cannot be resolved.

The accumulation of mutations in these inserts may also be a result of the new DIC application, if it has an inherently high mutation rate. This is not likely however, since all mutations were beyond the region of homology between the vector and insert, therefore the introduction of these mutations could not be due to mis-binding during the annealing stages of DIC. This suggests that the mutation was incorporated either during the elongation (gap-filling) stage of DIC or during amplification of the individual components. Based upon the reported high fidelity of the enzyme, we expect this to be a very rare event. It is more likely that DIC has an extremely high cloning efficiency and the toxicity associated with expression of these cassettes by the $\operatorname{sip} A$ promoter allowed for the recovery of those rare clones bearing inserts that have undergone mutation during construction. This would also explain why traditional cloning techniques were consistently unsuccessful. The optimal parameters for DIC have not yet been completely defined, but we are confident DIC will allow for cloning of inserts at a high enough efficiency to allow for direct transformation into Lactobacillus and thereby eliminate the 
requirement for $E$. coli as an intermediate cloning host and alleviate concerns associated with toxicity in this organism.

This investigation yielded the successful construction of a broad host range shuttle vector with a robust multiple cloning sequence allowing relatively easy cloning and expression of DNA in Lactobacillus and other organisms in which the pGK12 plasmid will replicate. This work also revealed toxicity of unknown origin that resulted in an inability to recover clones containing the intact PLGAT and PLGT inserts. Furthermore, we developed a novel cloning technique, which may allow for direct cloning of these cassettes into Lactobacillus and bypass the toxicity problem in E. coli. Until it is determined whether or not DIC can be used for this application, inducible expression systems for E. coli and Lactobacillus will be used prevent selection for mutants during cloning and allow for analysis of the slpA localization signals in both organisms. 


\subsection{Construction of inducible plasmids systems for \\ Lactobacillus and $E$. coli to determine the localization of prolyl peptidase activity under control of the slpA}

\section{localization signals.}

\subsection{Introduction}

As previously described, prolyl peptidases have been suggested as a potential treatment for Celiac disease due to the unique ability of these enzymes to hydrolyze immunogenic peptides associated with this autoimmune disease. Peptidases from the bacterium Myxococcus xanthus (MxPEP) as well as from the mold Aspergillus niger (ANPEP) are known to actively degrade the proline-rich peptides (Shan et al. 2004, Stepniak et al. 2006). While Lactobacillus containing cultures have been shown to slowly digest immunogenic peptides (Deangelis et al. 2006; Rizzello et al. 2007), the activity of individual peptidases expressed by members of the genus against the same peptides has not yet been investigated. The Lactobacillus genus contains three classes of proline specific peptidases widely reported in the literature. Prolinases (PepPN) are able to cleave proline diaminoacid (Pro-Xaa) substrates (Varmanen et al., 1998). Prolyl oligopeptidases of the S9 family of serine proteases (S9POP) are common in the Lactobacillus genus. Prolyl oligopeptidases generally have a predilection for peptides shorter than 30 amino acids and exhibit variable specificity for the cleavage site with respect to the amino acids surrounding proline residues (Polgar, 2002). Lastly, X-prolyl-diaminopeptidases (XPDAPs) can also be found throughout the genus. A point mutation in the coding region 
for this enzyme in L. helveticus has resulted dramatic growth inhibition in milk, which contains a large amount of proline-rich casein proteins (Yuksel \& Steele., 1996). Futhermore, some casein-derived digestion resistant peptides are rapidly degraded by these enzymes (Degraeve \& Martial-Gros, 2003). Finally, it has been shown that the one of two enzymes that limits the rate of degradation of the $\mathrm{CD}$ immunodominant epitopes in the intestine is the human XPDAP, dipeptidyl peptidase IV (DPPIV). Since all of these peptidases are primarily localized to the cytoplasm, a secretion signal would need to be incorporated to allow for extracellular expression and an anchoring domain may be desirable to tether the enzyme to the exterior cell wall.

The previous chapter showed that the expression and/or localization signals derived from the slpA gene of Lactobacillus acidophilus NCFM are toxic in E. coli, leading to the inability to recover clones bearing intact inserts. Inducible plasmid-based expression systems are often used to allow expression of inserts that may be toxic to their host. To effectively assess the efficacy of using $\operatorname{slp} A$ localization signals to guide surface layer expression of prolyl peptidase activity, inducible expression systems for both E. coli and Lactobacillus are necessary. These inducible systems would allow for the expression of the desired insert only after induction, thus preventing selection for mutants during cloning and allowing for the controlled assessment of $\operatorname{slp} A$ signals in both organisms.

In E. coli, tightly regulated expression systems have been designed based upon the regulatory features of the lac operon. A commercially available E. coli expression system, pET30, exploits the IPTG controlled expression of the lac operon to provide a dually regulated mechanism with strict but easily inducible expression of heterologous proteins. Two components are essential for the system: 1) a plasmid containing the $\mathrm{T}_{7}$ 
promoter under control of the lac operator; 2) a specialized host strain (BL21DE3) that expresses the $T_{7}$ RNA polymerase under control of the lac promoter and operator. This dual layer of regulation allows for very stringent control over the expression of the cloned sequence of interest.

To assess the effectiveness of S-layer derived expression cassettes in Lactobacillus species, an inducible expression system based on pGK12 was conceived. As previously described, the pGK12 shuttle plasmid contains an inducible promoter that controls the expression of the erythromycin resistance promoter. By replacing the ErmC open reading frame with the $\operatorname{slp} A$ fusion cassettes, an erythromycin inducible shuttle vector could be constructed.

The current investigation describes the utilization of pET30 based plasmids to assess the viability of using $\operatorname{slp} A$ signals to drive prolyl peptidase localization in E. coli. Also, described in this work is the construction of an erythromycin inducible shuttle vector, and evaluation of this plasmid's potential to control expression of toxic protein products in E. coli and Lactobacillus. 


\subsection{Materials and Methods}

3.2.1 Strains, plasmids and growth conditions

All plasmids were built and initially propagated in NEB Turbo E. coli. Cultures were grown in Luria Bertani broth or plates. When necessary ampicilin (100 $\mu \mathrm{g} / \mathrm{mL})$, chloramphenicol $(15 \mu \mathrm{g} / \mathrm{mL})$ and erythromycin $(250 \mu \mathrm{g} / \mathrm{mL})$ were added to the media to select for plasmid harboring strains. Shuttle plasmids were built in E. coli and then transformed into L. acidophilus according to the procedure described in chapter 2 (pg. 27) and propagated in Man-Rogosa Sharpe (MRS) broth or agar with chloramphenicol (5 $\mu \mathrm{g} / \mathrm{mL})$ or erythromycin $(5 \mu \mathrm{g} / \mathrm{mL})$. All plasmids used in this study are listed in Table 3-1. Myxососcus xanthus and Aspergillus niger were propagated in trypticase soy agar.

All molecular techniques used in this study were the same as in chapter 2 (pg. 24) The primers used in this study are listed in Table 3-2. 


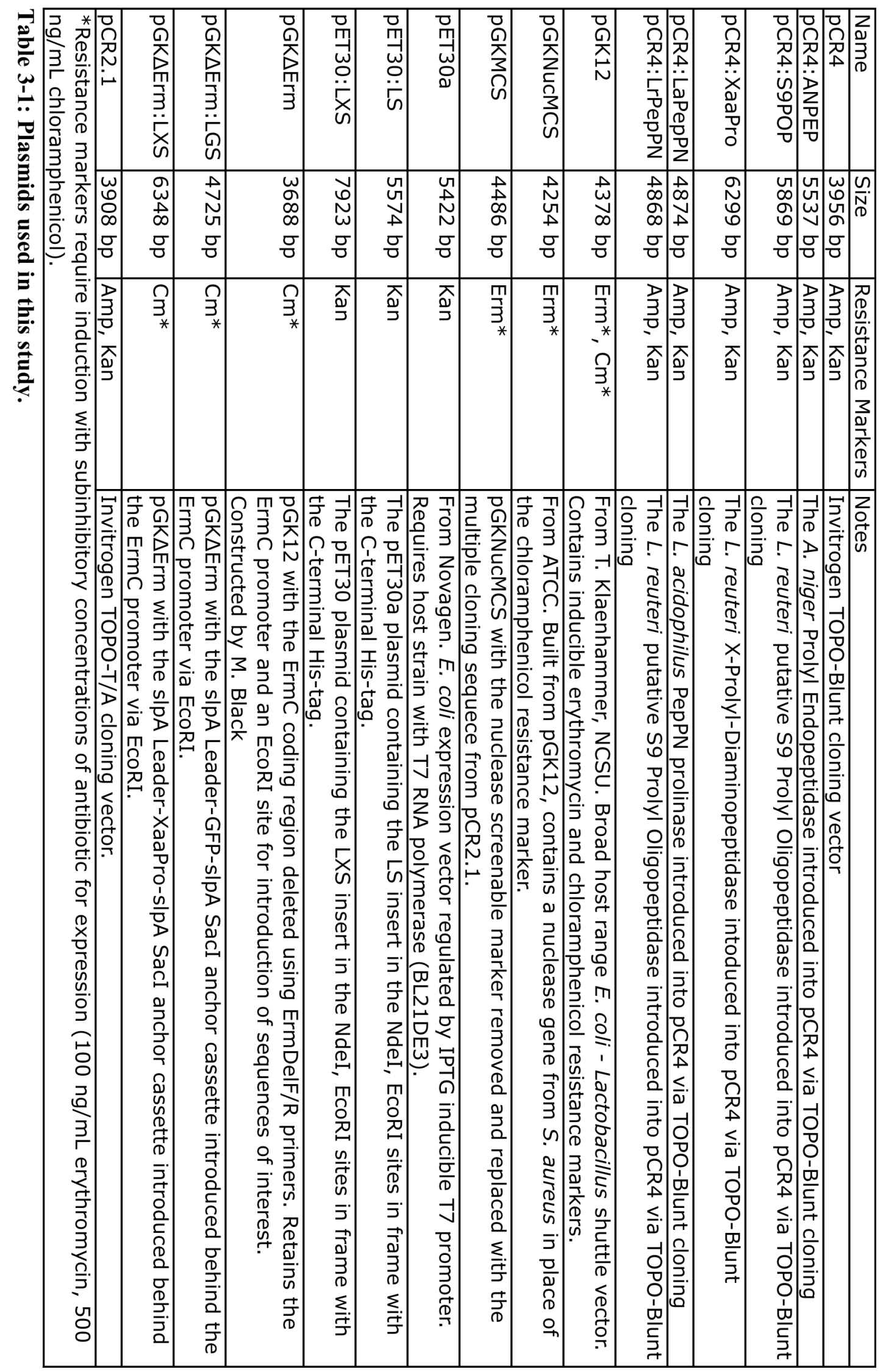



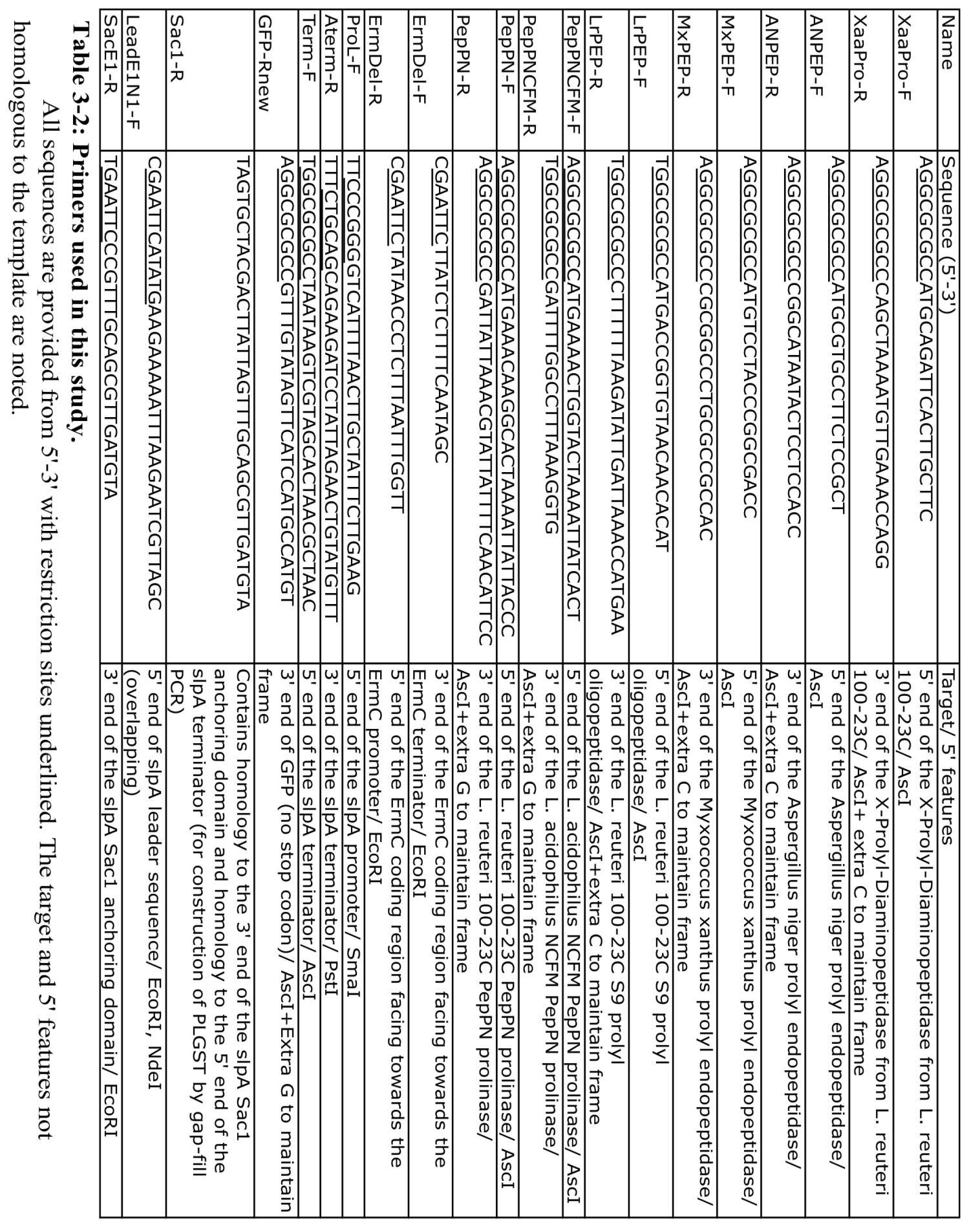


\subsubsection{Amplification and cloning of prolyl peptidases}

Prolyl endopeptidases from Aspergillus niger and Myxococcus xanthus have been described previously (Stepniak et al., 2006; Shan et al., 2004). Four peptidases from $L$. reuteri (S9 prolyl oligopeptidase-S9 POP, Xaa-Pro diamino peptidase- XPDAP, and proline iminopeptidase- LrPepPN) and L. acidophilus (proline iminopeptidaseLaPepPN) were selected for sub-cloning. Amplification of peptidases was performed with Phusion DNA polymerase using the appropriate primer pair listed in Table 3-2 and according to previously described thermal cycling parameters (pg 24). For all bacterial peptidases extracted genomic DNA (ZR Fungal/Bacterial DNA Kit, Zymo Research) was used as template for amplification. The $L$. reuteri peptidases were amplified from the genome of $L$. reuteri 100-23C and the L. acidophilus PepPN was amplified from the genome of L. acidophilus NCFM. The Myxococcus xanthus prolyl endopeptidase (MxPEP) was amplified from the genome of Myxococcus xanthus ATCC 25232. For Aspergillus niger total RNA was extracted using the RNeasy kit (Qiagen) and converted to cDNA using the Superscript cDNA synthesis kit (Invitrogen) according to manufacturers instructions. After cDNA synthesis ANPEP was amplified by Phusion DNA polymerase as previously described except the cycle number was increased to 40 . All amplified peptidases were sub-cloned into the TOPO-blunt vector pCR4 (Invitrogen) according to manufacturers instructions. 


\subsubsection{Construction of the erythromycin inducible expression vector $\mathrm{pGK} \Delta \mathrm{Erm}$}

pGK $\Delta$ Erm was incidentally built by $\mathrm{M}$. Black for a related thesis topic. Primers with $5^{\prime}$ EcoRI sites were designed from pGK12 to delete the open reading frame of ErmC but preserve the promoter and terminator sequences, as well as the remainder of the pGK12 backbone (Ermdel-F/R). The resulting amplicon was digested with EcoRI and ligated to re-circularize creating $\mathrm{pGK} \Delta \mathrm{Erm}$. After transformation, this plasmid was selected for using the remaining chloramphenical resistance marker.

\subsubsection{Construction of the PLGST cassette and amplification of LGS}

The Promoter-Leader-GFP-Sac1-Terminator cassette was built by gap-fill PCR. The Promoter-Leader-GFP, and 5' GFP seq-Sac1 and Term gene fusion products were amplified by PCR with the uncloned PLGAT amplicon as template and the appropriate primer pairs (ProL-F/GFP-R, 5' GFP seq/Sac1-R and Term-F/Aterm-R, respectively) using Phusion DNA polymerase. The Sac1-R primer contained 20 nucleotides of homology with the 5' end of the slp $A$ terminator. For gap-fill PCR, $2 \mu \mathrm{L}$ of each amplicon was mixed together in a $20 \mu \mathrm{L}$ Phusion reaction, the thermocycling parameters were as follows: $98^{\circ} \mathrm{C}$ for 1 minute 5 cycles of $98^{\circ} \mathrm{C}$ for 10 seconds, $60^{\circ} \mathrm{C}$ for 30 seconds and $72^{\circ} \mathrm{C}$ for $45 \mathrm{~s}$ and a final elongation at $72^{\circ} \mathrm{C}$ for 2 minutes. Immediately following the gap-fill reaction, $1 \mu \mathrm{L}$ of HF buffer, $1 \mu \mathrm{L}$ of $10 \mu \mathrm{M}$ ProL-DIC-F, $1 \mu \mathrm{L}$ of $10 \mu \mathrm{M}$ AtermDIC-R and $2 \mu \mathrm{L}$ of $\mathrm{H}_{2} \mathrm{O}$ was added and taken through PCR parameters as described previously for Phusion. The promoter-less and terminator-less Leader-GFP-Sac1 (LGS) gene fusion product was amplified from PLGST with LeadE1N1-F and Sac1E1-R 
primers to provide compatible restriction sites for introduction into pGK $\Delta$ Erm (via EcoRI) and pET30a (via NdeI and EcoRI).

\subsubsection{Construction of pGK $\Delta$ Erm:LGS, pGK $\Delta$ Erm:LXaaProS and pET30a:LXaaProS, pET30a:LS}

The LGS amplicon was digested with EcoRI and ligated into EcoRI linearized pGK $\Delta$ Erm to create pGK $\Delta$ Erm:LGS. Colony PCR was used to identify clones that had the introduced LGS cassette in the propero orientation to place it behind the ErmC promoter. The pGK $\Delta$ Erm:LXaaProS plasmid was created by digesting pGK $\Delta$ Erm:LGS with AscI to remove GFP then ligating AscI digested XaaPro from pCR4:XaaPro. After

plasmid propagation and extraction in E. coli, pGK $\Delta$ Erm:LGS, pGK $\Delta$ Erm:LXaaProS and pGK $\Delta$ Erm were transformed into L. acidophilus NCFM.

Since the XaaPro sequence contained two NdeI sites, a two-step cloning strategy was required to build pET30:LXS. The plasmid pET30:LS was constructed during the first step. The Leader-Sac1 (LS) cassette was amplified from the Promoter-Leader-Sac1Terminator (PLST) construct which was built by gap-fill PCR similar to PLGST (J. Labarge). $5 \mu \mathrm{L}$ of LS was then digested with NdeI in a $20 \mu \mathrm{L}$ reaction containing $2 \mu \mathrm{L} 10$ X EcoRI buffer, $1 \mu \mathrm{L}$ of NdeI and $11 \mu \mathrm{L} \mathrm{H}_{2} \mathrm{O}$ and incubated at $37^{\circ} \mathrm{C}$ for 1 hour. After the initial 1 hour incubation, $1 \mu \mathrm{L}$ of EcoRI was added and the reaction was incubated a further 1 hour at $37^{\circ} \mathrm{C}$ after which it was heat inactivated $\left(65^{\circ} \mathrm{C}\right.$ for 20 mins) and ligated into NdeI and EcoRI digested pET30a (Novagen) to form pET30a:LS. The 
pET30a:LXaaProS plasmid was built by linearizing pET30:LS with AscI and ligating in the gel purified AscI digested XaaPro sequence from pCR4:XaaPro.

3.2.6 Western blot analysis of inducible GFP expression from pGK $\Delta$ Erm:LGS E. coli bearing $\mathrm{pGK} \Delta \mathrm{Erm}: \mathrm{LGS}$ and $\mathrm{pGK} \Delta \mathrm{Erm}$ (negative control) were grown in LB-Chloramphenicol $(15 \mu \mathrm{g} / \mathrm{mL})$ to $\mathrm{OD}_{600}=0.5$ then induced for two hours with 10 $\mathrm{ng} / \mathrm{mL}$ erythromycin. Uninduced controls were grown without induction for two hours. Three $\mathrm{OD}_{600}$ units (i.e. $6 \mathrm{~mL} @ \mathrm{OD}_{600}=0.5$ ) was harvested at the 0 minute induction timepoint and the two hour induction time point. The samples were centrifuged at $4000 \mathrm{Xg}$ for 10 minutes at $4^{\circ} \mathrm{C}$ and $1 \mathrm{~mL}$ of the media was removed and stored at $-80^{\circ} \mathrm{C}$ (Media fraction). The remaining media was discarded and the cells were washed $1 \mathrm{X}$ with PBS $\mathrm{pH}$ 7.4, pelleted and snap frozen in liquid nitrogen and stored at $-80^{\circ} \mathrm{C}$. At the time of processing, the cells were thawed and resuspended in $500 \mu \mathrm{L}$ PBS $+0.1 \%$ Triton X-100 and lysed by sonication for $2 \mathrm{X}$ for $10 \mathrm{~s}$ at power setting 5 ( 14 RMS). The insoluble portion of the lysate was pelleted and the supernatant (Lysate fraction) was removed and stored at $-80^{\circ} \mathrm{C}$. The remaining pellet (Pellet fraction) was also stored $@-80^{\circ} \mathrm{C}$. AntiGFP western blot was performed according to the protocol on pg. 35. In each case the volumes were normalized so as to load an equal volume relative to the original sample of each fraction into each lane. 


\subsubsection{His-tag detection of pET30 expressed proteins}

The pET30:LS and pET30:LXS plasmids, were transformed into E. coli BL21DE3 (Novagen). Overnight cultures in LB-Kanamycin $(10 \mu \mathrm{g} / \mathrm{mL})$ were diluted 100 -fold in the same medium and grown to $\mathrm{OD}_{600}=0.5$, at which point each culture was induced for two hours with $1 \mathrm{mM} \mathrm{IPTG}$ at $37^{\circ} \mathrm{C}$ with shaking. The media, lysate and pellet fractions were collected as described above. Detection of full-length protein expression was accomplished using an HRP conjugated His Probe (Pierce), which binds to the His-tag and can visualized using the ECL + detection reagent (Amersham Biosciences). The His-tag detection and visualization was carried out according to the manufacturers instructions.

\subsubsection{Growth curves analysis of pET30 plasmids}

E. coli BL21DE3 cells bearing the pET30:LS and pET30:LXS plasmids were cultured overnight in LB-Kanamycin $(10 \mu \mathrm{g} / \mathrm{mL})$, diluted 100 -fold in the same medium then grown to $\mathrm{OD}_{600}=0.5$ and induced as described above. $\mathrm{OD}_{600}$ readings were taken at the time of induction ( 0 time point) and then every 15 minutes for 2 hours. Uninduced controls were used for microscopic comparison. 


\subsubsection{Prolyl peptidase activity assays}

E. coli BL21DE3 harboring pET30:LXS and NEB Turbo E. coli with

pGK $\Delta$ Erm:LXS or pGK $\Delta$ Erm (negative control) were grown overnight in the appropriate selective media. The cultures were diluted 100 -fold and grown to $\mathrm{OD}_{600}=0.5$ and induced for two hours with either 1mM IPTG (pET30:LXS) or $10 \mathrm{ng} / \mathrm{mL}$ erythromycin (pGK $\Delta$ Erm:LXS and pGK $\Delta$ Erm). Whole cell lysates were prepared as described above with the exceptions that the cells were sonicated in $500 \mu \mathrm{L}$ of $1 \mathrm{mM}$ Tris- $\mathrm{HCl}(\mathrm{pH} 8.0)$ and the insoluble material was not pelleted after lysis. Intact cell samples were harvested by centrifugation, washed one time in $1 \mathrm{mM}$ Tris- $\mathrm{HCl} \mathrm{pH} 8.0$ and resuspended in $500 \mu \mathrm{L}$ of $1 \mathrm{mM}$ Tris-HCl pH 8.0.

Lactobacillus NCFM harboring pGK $\Delta$ Erm and pGK $\Delta$ Erm:LXS were grown to $\mathrm{OD}_{600}=0.5$ and harvested after two hours of induction with $10 \mathrm{ng} / \mathrm{mL}$ erythromycin. Whole cell lysates were prepared by resuspending in $500 \mu \mathrm{L}$ of $\mathrm{H}_{2} \mathrm{O}$ and adding 100 units/mL of mutanolysin and $100 \mu \mathrm{g} / \mathrm{mL}$ of lysozyme and incubating for 30 minutes @ $37^{\circ} \mathrm{C}$. After protoplasting, the cells were lysed by the same sonication procedure as was used for E. coli and lysis was confirmed by microscopy.

Peptidase assays were performed in a 96 well flat bottom optical plate (Corning) with a $200 \mu \mathrm{L}$ reaction volume. Each assay consisted of $180 \mu \mathrm{L}$ of sample (whole cell lysate, intact cells or supernatant) and $20 \mu \mathrm{L}$ of $100 \mathrm{mM}$ chromogenic substrate Ala-PropNA or Gly-Pro-pNA (Bachem). Blanks consisted of $20 \mu \mathrm{L}$ of substrate with $180 \mu \mathrm{L}$ of either $1 \mathrm{mM}$ Tris-HCl, LB or MRS. Enzyme activity was monitored by detecting absorbance $(\lambda=405 \mathrm{~nm})$ every 2 minutes for 20 minutes at $37^{\circ} \mathrm{C}$. 


\subsection{Results}

\subsubsection{Sub-cloning of peptidases into pCR4}

Aspergillus niger prolyl endopeptidase (ANPEP), Myxococcus xanthus prolyl endopeptidase (MxPEP), S9 prolyl oligopeptidase (S9 POP), X-prolyl diamino peptidase (Xaa-Pro), L. reuteri PepPN (LrPepPN), and L. acidophilus (LaPepPN) were all successfully amplified using the appropriate primer pair and template. Sub-cloning into pCR4 was successful for five of the six peptidases of interest. In each case, cloning was confirmed diagnostic digest of the plasmids with AscI (Figure 3-1). Cloning of the gel purified MxPEP sequence was unsuccessful in three separate TOPO-Blunt ligations. 


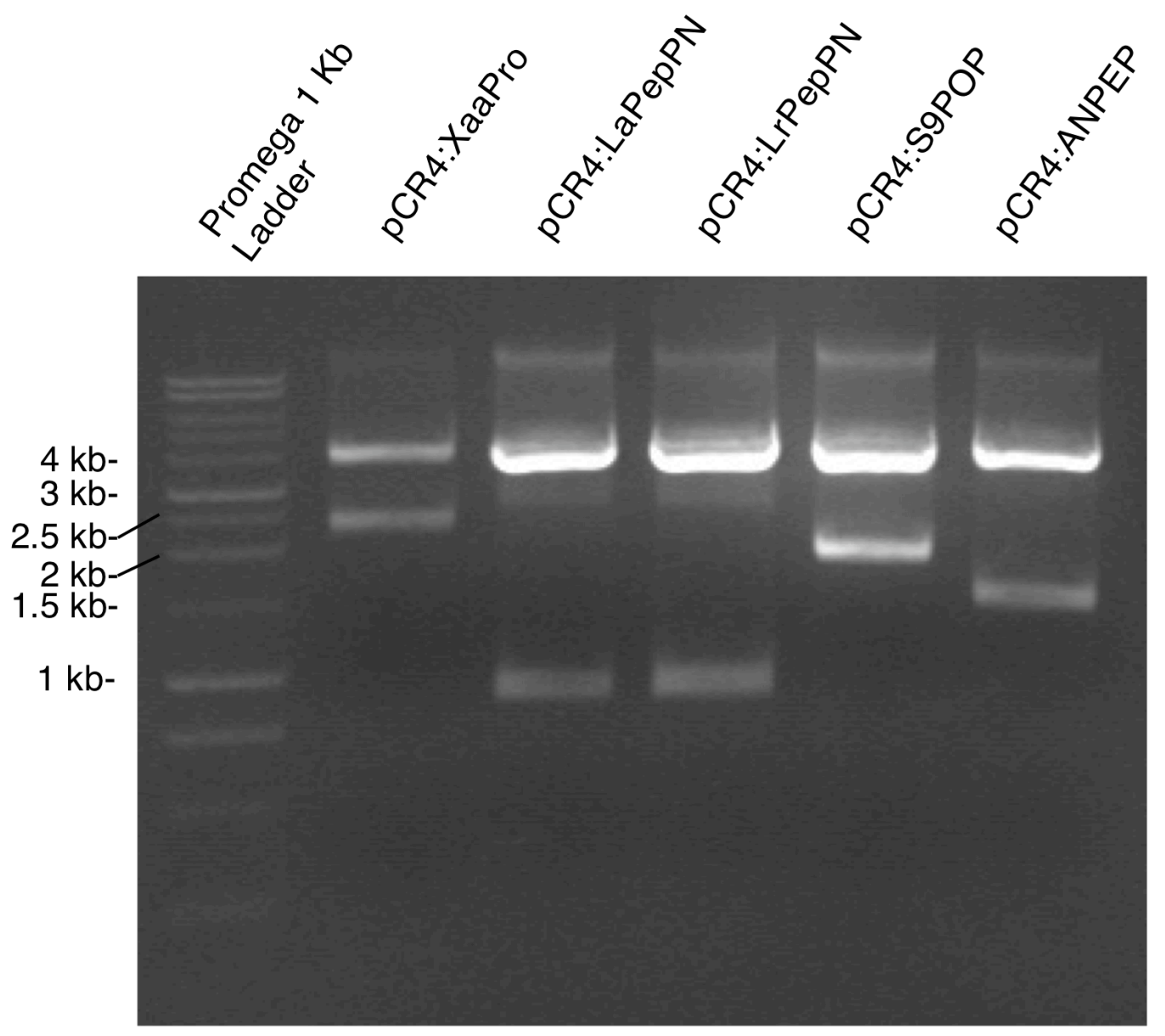

Figure 3-1: Cloning of prolyl peptidases into the pCR4 TOPOblunt vector.

Each plasmid was digested with AscI to remove cloned sequence and analyzed by agarose (1.5\%) gel electrophoresis. Expected sizes: $\mathrm{pCR} 4=3956 \mathrm{bp}$, XaaPro $=2343 \mathrm{bp}, \mathrm{LaPepPN}=918$ bp, LrPepPN = 912 bp, S9POP = 1913 bp, ANPEP = 1581 bp.

\subsubsection{Characterization of the erythromycin inducible plasmid pGK $\Delta$ Erm:LGS}

An initial assay with $10 \mathrm{ng} / \mathrm{mL}$ erythromycin showed an increase in fluorescence after 30 minutes of induction (Fig 3-2). Longer incubation times resulted in lower GFP expression (data not shown). Instability of pGK $\Delta$ Erm:LGS made long term maintenance of this plasmid in E. coli impossible. Preparation of plasmid DNA from successive cultures of $E$. coli harboring pGK $\Delta$ Erm:LGS, showed a gradual shift from the intact 
plasmid to a smaller plasmid of unknown composition. This cryptic plasmid was linearized by EcoRI without removing a band corresponding to LGS, the linearized plasmid was smaller than the parental vector, pGK $\Delta$ Erm. After the selected clone was found to be unstable, another clone that was positive by PCR was cultivated. This clone showed no evidence of plasmid instability. However, this clone also showed no evidence of GFP expression by either fluorescence microscopy (data not shown) or western blot analysis (Figure 3-3) when compared to negative controls transformed with pGK $\Delta$ Erm.

Only the stable and non-fluorescent clone of pGK $\Delta$ Erm:LGS was successfully introduced into Lactobacillus NCFM. The resulting clones were confirmed to contain the intact plasmid by extraction and diagnostic digest with AscI. The transformed Lactobacillus strain showed did not evidence of inducible GFP expression when compared to strains harboring pGK $\Delta$ Erm (data not shown). 

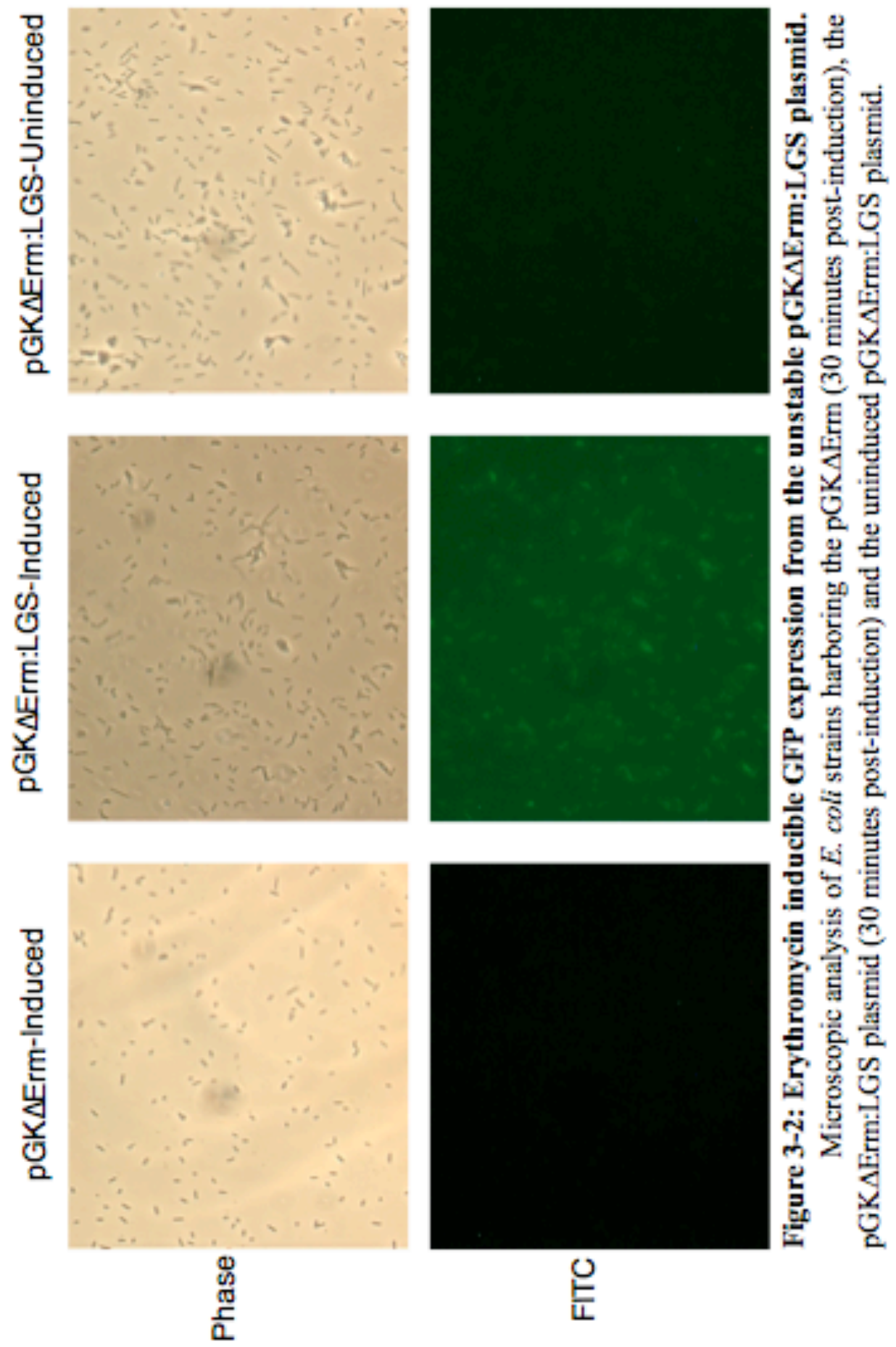

맹 
a) pGK $\Delta$ Erm:LGS
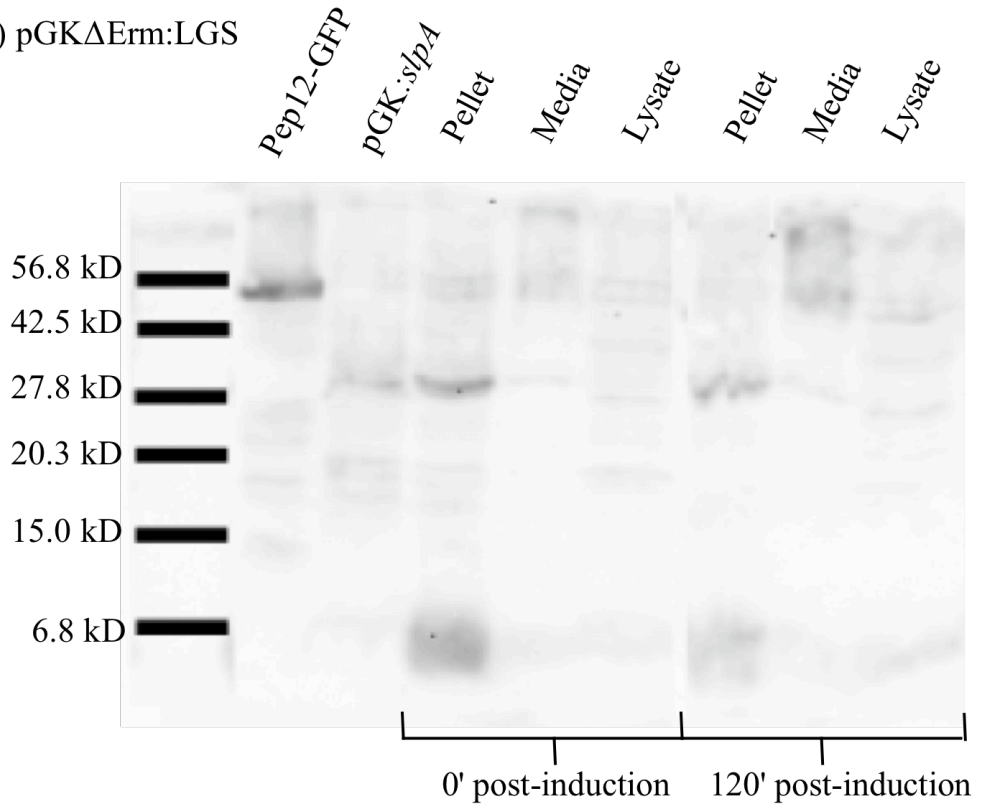

b) pGK $\Delta \mathrm{Erm}$
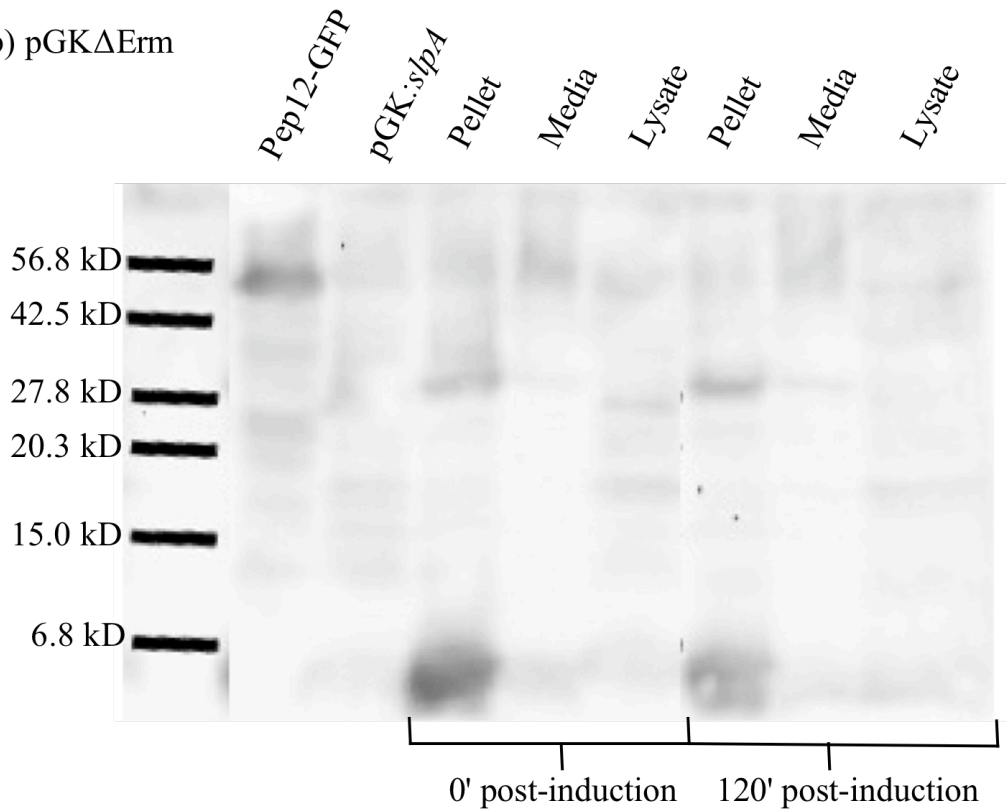

Figure 3-3: Western blot analysis of inducible GFP expression in the stable pGKAErm:LGS plasmid.

Anti-GFP western blot of $E$. coli strains carrying a) pGK $\Delta$ Erm:LGS and b) pGK $\Delta$ Erm (negative control) at 0 minutes and 120 minutes post-induction with $10 \mathrm{ng} / \mathrm{mL}$ of erythromycin. For each trial the same relative amount of cell pellet, media and lysate was loaded to confirm the absence of GFP expression in all fractions. The expected size for LGS is $37.7 \mathrm{kD}$. Controls for each blot included Pep12-GFP fusion (positive control) and a whole cell lysate from uninduced $E$. coli harboring pGK:slpA (negative control). 
3.3.3 Utilization of the pET30 expression system to determine the source of toxicity and peptidase localization in E. coli.

Two pET30 based plasmids, pET30:LXS and pET30:LS were built in order to determine the localization of peptidase expression in E. coli. To determine if toxicity was associated with either insert, growth was monitored after induction with IPTG. The pET30:LS plasmid showed essentially no growth after 15 minutes and even a slight drop in OD600 after 1.5 hours post-induction. Upon induction, pET30:LXS exhibited only a small degree of growth impairment when compared to the uninduced control (Figure 3-

4).

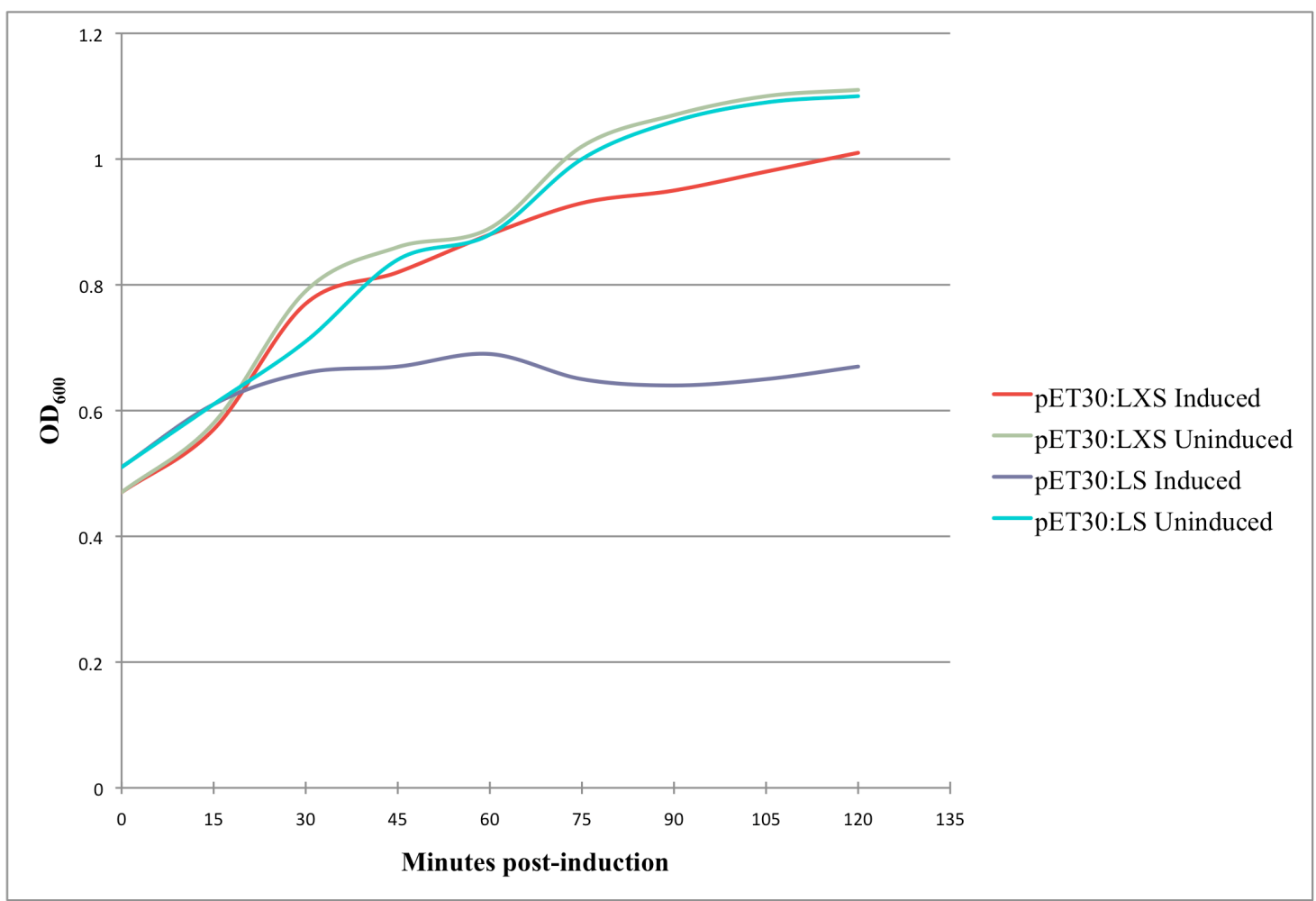

Figure 3-4: pET30:LXS and pET30:LS growth curves

Growth curves from E. coli BL21DE3 cells harboring the pET30:LXS or pET30:LS plasmids. OD 600 measurements were taken every 15 minutes from 0 minutes to 120 minutes post induction with $1 \mathrm{mM}$ IPTG or without induction. 
The two pET30 plasmids were designed to preserve the downstream His-tag as part of the inducible open reading frame, thus allowing for determination of full length insert expression by detection of the His-tag using an HRP conjugated His Probe. The His-probe analysis showed full-length LXS expression $(101.1 \mathrm{kD})$ localized to the insoluble pellet fraction of the cell lysate. Expression of LS $(12.7 \mathrm{kD})$ was much lower and was also localized to the pellet fraction (Figure 3-5). No peptidase activity could be detected in whole cell lysates prepared from the induced pET30:LXS cultures despite the evidence of full-length expression of the insert by His-tag detection, perhaps suggesting that the LXS protein is being expressed in inclusion bodies (data not shown).

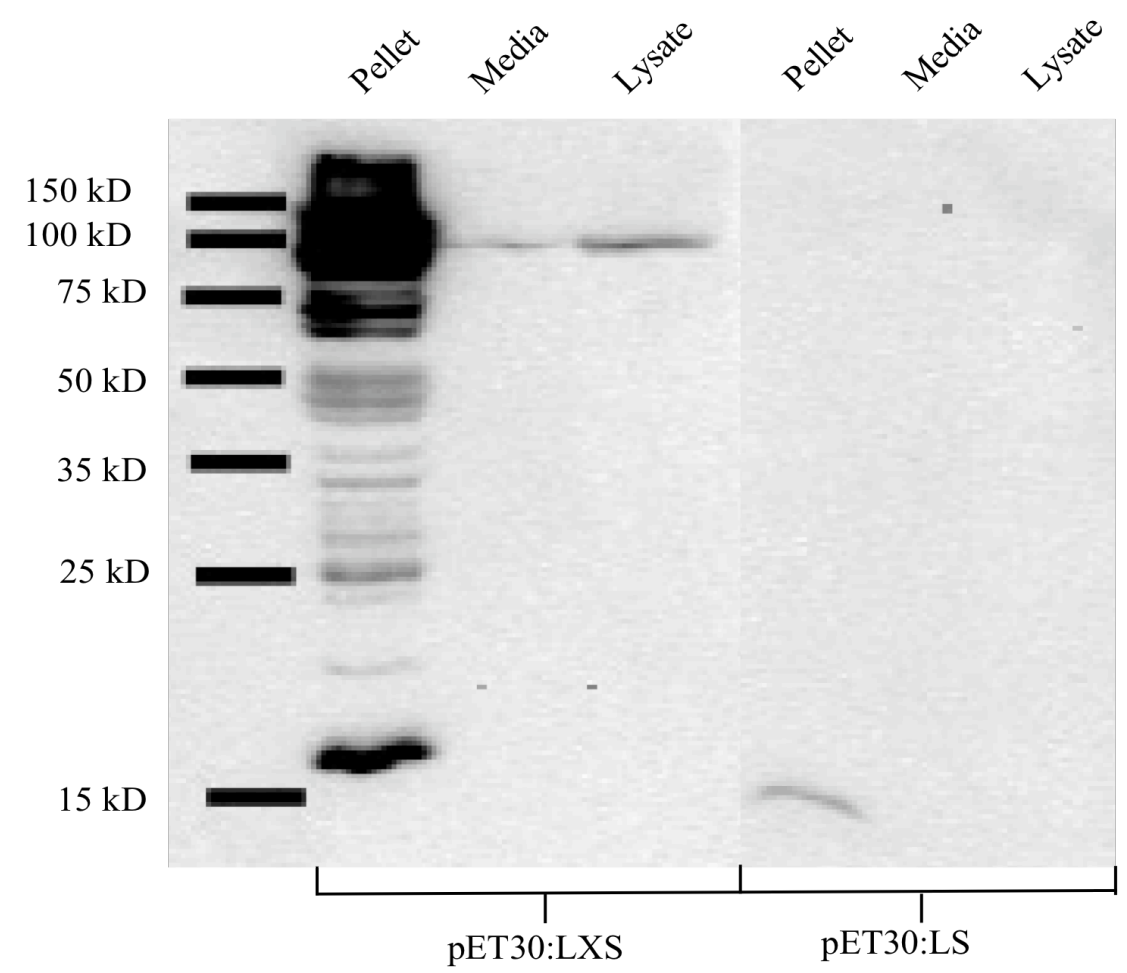

Figure 3-5: Localization of LXS and LS in E. coli.

E. coli BL21DE3 cells harboring pET30:LXS and pET30:LS were grown to $\mathrm{OD}_{600}$ and induced for 2 hours with $1 \mathrm{mM} \mathrm{IPTG}$. The C-terminal His tag was detected using the HRP conjugated His probe. Equal relative volumes from each fraction were loaded in order to determine localization. The expected sizes for LXS and LS were $101.1 \mathrm{kD}$ and $12.7 \mathrm{kD}$ respectively. 
To determine if inclusion body formation was a possibility, cultures were observed by microscopy. Distinctive morphological differences were observed between induced cultures of E. coli harboring pET30:LXS and uninduced cultures. Induced cultures showed drastically elongated cellular morphology with irregular coloration. Uninduced cultures as well as the induced and uninduced pET30:LS control cultures displayed the individual $1 \mu \mathrm{m}$ by $2 \mu \mathrm{m}$ rods characteristic of $E$. coli (Figure 3-6).

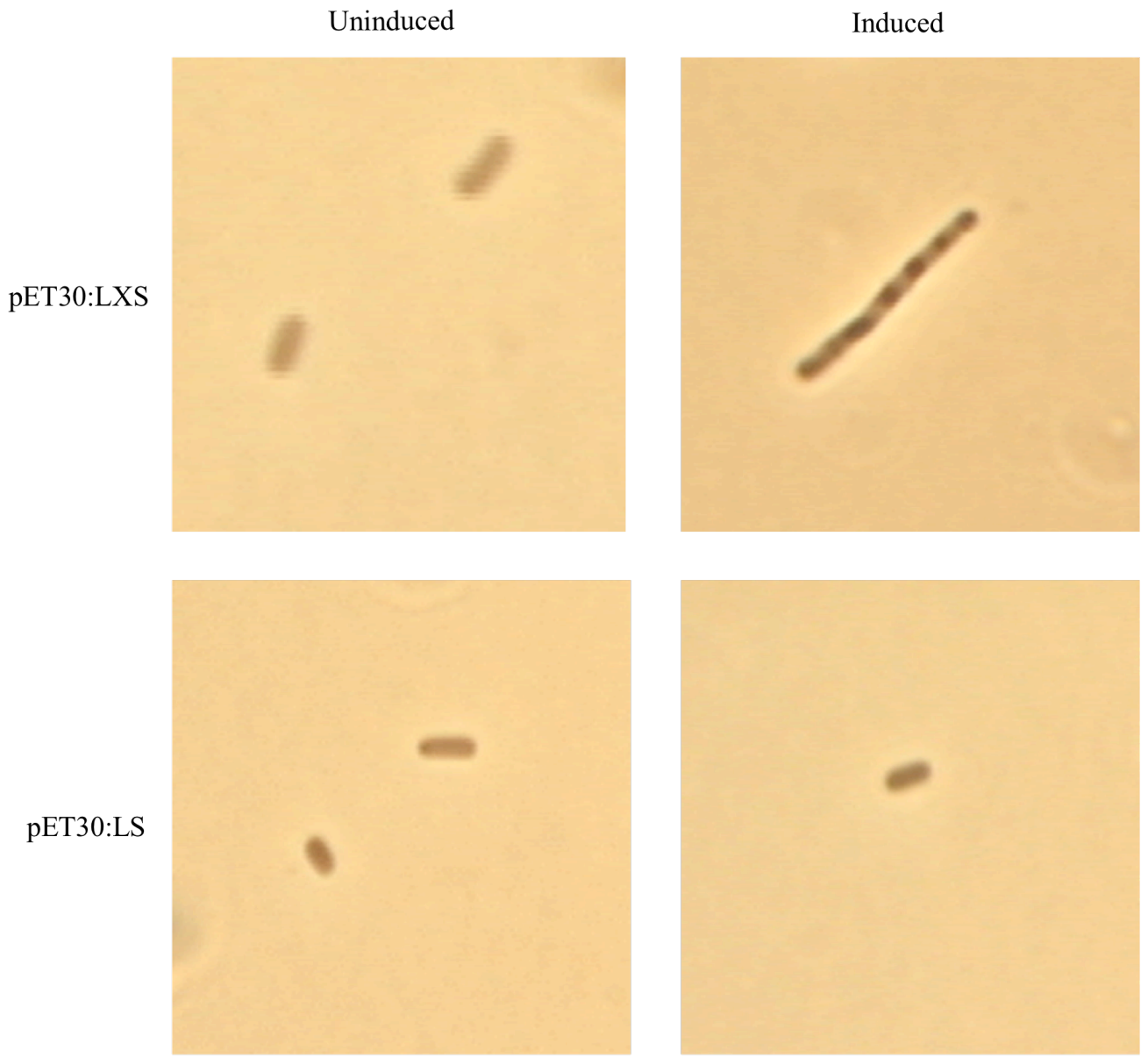

Figure 3-6: Cellular morphology of induced and uninduced pET30:LS and pET30:LXS cultures Induced and uninduced E. coli BL21DE3 cells with pET30:LS or pET30:LXS were visualized by phase contrast microscopy (1000X total magnification). Fields of view were chosen to accurately represent the dominant morphologies observed in each population. 


\subsection{Discussion}

The first stage of this investigation involved the selection, amplification and subcloning of peptidases of interest into the TOPO-Blunt vector pCR4. Two peptidases known to degrade celiac associated peptides, MxPEP and ANPEP, were chosen to act as positive controls for future experiments. Only ANPEP was successfully cloned and this was only after the PCR product of the expected size was gel purified away from smaller background products. Cloning of the MxPEP peptidase into pCR4 was not successful. The MxPEP amplification also showed a great deal of background amplification. After gel purification the expected sized band was faint. This lack of product yield is the likely reason for the inability to recover pCR4 clones containing the MxPEP insert.

Enzymes from each of the three classes of prolyl peptidases expressed in the Lactobacillus genus were successfully amplified and sub-cloned into pCR4 (Figure 3-1). These peptidases can be shuttled into an expression vector utilizing the engineered AscI sites flanking each open reading frame. To assess enzyme activity in the context of the $\operatorname{slp} A$ localization signals, inducible expression systems had to be utilized.

Expression of the ErmC protein is regulated by an inducible promoter that requires a subinhibitory concentration of erythromycin for efficient translation of the gene product. It was then reasoned that an adequate inducible E. coli-Lactobacillus shuttle vector could be constructed by replacing the ErmC coding region with the LGS cassette. Although, fluorescence assays indicated the induction of GFP expression compared to the uninduced control, the plasmid appeared to be unstable and could not be maintained. Evidence of a recombination event that removed the LGS cassette along with 
a portion of the pGK12 backbone was noted. Since this selection took place in uninduced cultures, there is likely leakiness associated with this promoter. Leaky regulation would allow for low-level expression of the toxic insert, leading to a high degree of selection for mutants. Due to the observed instability and possible leakiness associated with the pGK $\Delta$ Erm:LGS shuttle plasmid it was concluded that this vector was not a viable option for inducible expression in E. coli or Lactobacillus. Since proper secondary structure of the 5' UTR and leader peptide are so critical for induction of the ErmC promoter, it is worth investigating whether or not the addition of the first 50 or so nucleotides at the 5 , end of the ErmC coding region to the cassettes of interest would result in more tightly regulated expression.

Due to the apparent failure in constructing an inducible pGK12-derived shuttle vector, the decision was made to switch to the better-characterized pET30 vector to regulate expression of the cassettes. The pET30:LS plasmid played a dual role in this investigation: (1) As a negative control to be used in the pET30:LXS peptidase activity assays. (2) Assessed toxicity associated with the S-layer localization components. Upon induction with IPTG, growth of the pET30:LS culture was abruptly halted. This observation provides undeniable evidence that the $\operatorname{slp} A$ leader and/or Sac1 anchor are toxic to $E$. coli. Although an attempt was made to remove any possible murein hydrolase activity from the anchor, the observed growth characteristics are consistent with the continued expression of this enzyme. Cell division in E. coli is partially facilitated by a careful balance between murein synthesizing and hydrolyzing enzymes in the periplasmic space between the cell membrane and outer envelope (Hakenbeck \& Messer, 1977). A shift in this equilibrium due to the catalytic activity prescribed to the LS fusion protein 
might result in an inability of the cells to grow and divide, thus explaining the growth cessation in Figure 3-4.

It was interesting to observe the distinctive difference in toxicity between the LS insert and the LXS insert. Since the His-probe showed full-length expression of the LXS cassette (Figure 3-5), two possible explanations remained for the attenuated toxicity associated with LXS as compared to LS.

First, LXS may be aggregating to form inclusion bodies in the cytoplasm of $E$. coli, thus is not able to elicit its toxic effects in the periplasmic space. This hypothesis may be supported by observed differences in cellular morphology between induced and uninduced LXS cultures. The induced culture showed drastically different morphology and contained what may be inclusion bodies within the cells. Meanwhile the induced LS cells showed no morphological differences from the uninduced control (Figure 3-6). The His-tag probe results may also support this assertion, since the vast majority of protein was associated with the insoluble cell lysate pellet. By definition, inclusion bodies are comprised of insoluble protein aggregates and would therefore localize to the pellet fraction after cell lysis and centrifugation. An alternative explanation for the presence of the expressed protein in the insoluble fraction is that the Sac1 anchor is functioning properly in E. coli and is anchoring to teichoic acid within peptidoglycan layer. If the protein is being expressed as an inclusion body the enzyme may still be able to be purified, re-solubilized and re-folded to determine enzymatic activity. Also, the concentration of IPTG used for induction could be dropped in order to achieve a level of induction that is not high enough to promote aggregation. 
Second, the attenuated toxicity may be a side effect of the introduction of the XaaPro cassette. In this scenario, the Xaa-Pro domain could abolish toxicity associated with the localization signals. The peptidase domain of the fusion protein may result in the inability of the leader peptide to be recognized or properly processed in E. coli leading to lack of secretion, which may ease the toxic affects of the insert. Likewise, if toxicity is associated with the Sac1 anchor, then the peptidase fusion may result in a conformation that abrogates any catalytic activity associated with this domain, thus leading to attenuated toxicity when compared to LS. At this time, the influence of the Xaa-Pro peptidase on toxicity has not been determined.

To properly assess the source of toxicity in E. coli (Leader or Sac1 or both), new plasmids allowing for every possible permutation of these components, are being built. These new plasmids (pET30:LX, XS, X, L, and S) may also provide insight into the reason why there is no Xaa-Pro peptidase activity detectable in induced pET30:LXS whole cell lysates. The lack of peptidase activity may be a result of at least two possibilities. The peptidase domain may not be able to fold correctly when fused to the Leader and/or Sac1 components, and /or this protein may require cellular components not present in E. coli that are required for protein folding or enzymatic activity. Furthermore microscopic and His probe analysis suggest the induced expression of LXS forms inclusion bodies, which are unlikely to show activity. Construction and analysis of the aforementioned vectors will provide understanding into what exactly is curtailing peptidase activity in the present study and may elucidate a solution to this problem.

Further work is required to answer the essential questions raised by this preliminary investigation: Are any of the selected Lactobacillus peptidases capable of 
hydrolyzing the immunodominant peptides associated with celiac disease? Can the $\operatorname{sip} A$ secretion and anchoring signals be utilized to drive the localization of these peptidases into the peptidoglycan layer of the chosen model or host organism? These questions will not be answered quickly or easily, but will require a step wise progression through organisms beginning with E. coli then proceeding to the model Lactobacillus acidophilus and eventually culminating in the construction of a final product within the target, $L$. reuteri. 


\subsection{Concluding remarks and future research}

The work described herein provides a solid technological foundation for the genetic engineering of lactobacilli. Among these advancements is the construction of an E. coli-Lactobacillus shuttle vector with a robust multiple cloning sequence as well as general procedures describing molecular techniques optimized for these bacteria. A notable achievement is the design of a novel method of digestion independent cloning (DIC), which once fully developed may allow for direct cloning of cassettes into Lactobacillus. This research also highlighted the unique difficulties inherent in nonmodel systems. Incompatibility between the Lactobacillus derived localization components and the $E$. coli cloning host in this study resulted in the inability to recover the desired shuttle plasmids and resulted in lost time and resources. To maximize the likelihood of future success, a three stage experimental plan has been developed.

Stage 1: Characterization of all components in the E. coli model. This initial investigation will allow for us to screen for prolyl peptidases that effectively hydrolyze immunogenic peptides when fused to secretion and anchoring signals. During this stage numerous pET30 vectors will be built to further characterize the source of toxicity observed in this study as well as the ability of each peptidase to function while fused to the localization signals. The peptidase activity screen will also be optimized during this stage and perhaps a broader range of peptidases will be studied to maximize the prospect of finding an enzyme that can be secreted and remain highly functional.

Stage 2: Determination of the functionality of S-layer localization components in the intermediate host, L. acidophilus. This stage utilizes the organism from which the S- 
layer components were originally isolated to determine if they are still functional when expressed in recombinant form and fused to the peptidase. A strain that does not express SlpA has also been obtained for this stage to nullify any concern of interference with localization due to competition with the native protein. For this stage, a stable inducible shuttle plasmid would be ideal. New inducible systems will be investigated (proteins involved in stress response in Lactobacillus have been proposed) and the ErmC promoter- based expression vector used in this work will be revisited in hopes of tightening regulation. Also, during this stage high-level and low-level constitutive promoters will be used to determine the level of expression the cell can tolerate. Here functionality will be tested, it will be essential to find the right combination of expression signals, localization signals and peptidase to allow proper expression in this host before moving on to the target organism.

Stage 3: Assess the efficacy in the final host organism, L. reuteri. During the final stage, proper surface level expression of peptidase activity will be appraised and enymatic assays will be performed in vitro and in vivo to determine if the peptidase delivery system is a viable treatment option for $\mathrm{CD}$. In depth analysis of enzymatic activity against the immunodominant peptides will be assessed during this stage. Not only rates of hydrolysis but also careful study of the digestion products using HPLC and LC-MS will be critical to ensure that the epitopes are being destroyed. In vitro analysis will be complemented with animal models (mice and pigs?) and immunological experiments (T-cell proliferation assays) to determine whether or not the delivery system can function in vivo. During this exciting final stage, translational questions will finally be addressed. 
By following this rough experimental design, future researchers are far more likely to and prevent long, wasteful expenditures of time and money. In this investigation, it is likely that the toxicity witnessed in the cloning host is a result of fundamental differences between the cellular physiologies of these organisms that are not yet completely understood. Indeed the exact function of the SlpA protein in Lactobacillus acidophilus has not yet been completely defined. This lack of knowledge underscores a fundamental limitation imposed by the nature of applied genetic research. The genomic revolution has provided an incredible wealth of information from a vast number of organisms with extremely diverse ancestry. While sequences are readily available and predictions can be made as to the function of gene products, their physiological roles often remain shrouded. The challenge of sifting through information and effectively mining the data for products that may be of therapeutic importance is laid upon the shoulders of researchers in the post-genomic era. The step-wise approach described above provides a framework for investigators in this field. While the exact stages will differ depending on the specific goal, it is important to be able to move through models to prevent the over-pursuance of false leads and maintain a steady course to the final goal.

Our research aims to create an entirely new form of treatment, not only for celiac disease but for other disorders in which enzyme supplementation through commensal microorganisms might be useful. This goal represents a paradigm shift in the way scientists view the human body. It is no longer seen as a collection of discrete parts that make up a whole, but as a single, integrated biological system in which microorganisms play a fundamental role. It is the challenge of future investigators to view the human body as a complex and interconnected system that is dependent not only on its own 
defenses and repair mechanisms but also the ecological community of its surfaces for optimal health and performance. This approach to the human body will require openmindedness, a broad background and interdisciplinary collaboration among a diverse array of scientific fields, but may provide the greatest prospect for elucidating a successful treatment for some of the most complicated and least manageable diseases. The beneficial role that bacteria play in human health remains relatively undefined. One must remember that the millions of years of human evolution did not happen in a vacuum. Just as bacteria have adapted to us, we have adapted to their presence not only around us but also within us. 


\section{Bilbiography}

Acosta MP., Palomina MM., Allievi MC., Riva CS., Ruzal SM. (2008) Murein Hydrolase Activity in the Surface Layer of Lactobacillus acidophilus ATCC 4356. Applied and Environmental Microbiology 74:7824-7827.

Anderson RP., Degano P., Godkin, AJ., Jewell DP and Hill AV. (2000) In vivo antigen challenge in celiac disease identifies a single transglutaminase-modified peptide as the dominant A-gliadin T-cell epitope. Nat Med 6: 337-342.

Ahrne S., Molin G., Axelsson L. (1992) Tranformation of Lactobacillus reuteri with electroporation: Studies on the erythromycin resistance plasmid pLUL631. Current Microbiology 24:199-205.

Arentz-Hansen H, Korner R, Molberg O, Quarsten H, Vader W, Kooy YM, Lundin KE, Koning F, Roepstorff P, Sollid LM, and McAdam SN. (2000) The intestinal T cell response to alpha-gliadin in adult celiac disease is focused on a single deamidated glutamine targeted by tissue transglutaminase. J ExpMed 191: 603-612.

Atkins JF., Elseviers D., Gorini L,. (1972) Low activity of B-galactosidase in frameshift mutants of Escherichia coli. PNAS 69:1192-1195

Boot HJ., Kolen C., van Noort J., Pouwels PH. (1993) S-layer Protein of Lactobacillus acidophilus ATCC 4356 purification, expression E. coli, and nucleotide sequence of the corresponding gene. J Bacteriology 6089-6096.

Boot HJ., Kolen C., Fotini, JA., Leer RJ., Pouwels PH. (1996) The Lactobacillus acidophilus S-layer protein gene site expression site comprises two consensus promoter sequences, one of which directs transcription of stable mRNA. $J$ Bacteriology 5388-5394.

Narayanan CS., Dubnau D. (1987) Demonstration of erythromycin-dependent stalling of ribosomes on the ermC leader transcript. J Biological Chemistry 262: 1766-1771.

Ciclitira, P., Johnson, M., Dewar, D., Ellis, J. (2005) The pathogenesis of celiac disease. Molecular Aspect of Medicine 26; 421-458.

Collin P., Pukkala E., Reunala T. (1996) Malignancy and survival in dermatitis herpetiformis: a comparison with coeliac disease. Gut 38: 528-530

Corazza GR., Brusco G., Anderani ML., Biagi F., Steffano MD.,Gasbarrini G. (1996) Previous misdiagnosis and diagnostic delay in adult celiac sprue. J Clinical Gasteroenterology 22: 324-325

Corrao, G., Corrazza, G.R., Bagnardi, V., Brusco, G., Giacci, C., Cottone, M., Sategna Guidetti, C., Usai, P., Cesari, G., Pelli, M.A., Loperfido, S., Volta, U., Calabro, A., Certo, M., (2001) Mortality in patients with coeliac disease and their relatives: a cohort study. Lancet 358; 356-361.

De Angelis M, Rizzello CG, Fasano A, Clemente MG, De Simone C, et al. (2006) VSL\#3 probiotic preparation has the capacity to hydrolyze gliadin polypeptides responsible for Celiac Sprue. Biochim Biophys Acta 1762: 80-93.

Degraeve, P., Martial-Gros, A. (2003) Purification and partial characterization of Xprolyl dipeptidly aminopeptidase of Lactobacillus helveticus ITG LH1. International Dairy Journal 13:497-507. 
De Vrese, M., Stegelmann, A., Richter, B., Fenselau, S., Laue, C. and Schrezenmeir, J. (2001) Probiotics-compensation for lactase insufficiency. American J Clinical Nutrition 73:421-429.

Dietrich, W., Ehnis, T., Bauer, M., Donner, P., Volta, U., Riecken, E.O., Schuppan, D., (1997) Identification of tissue transglutaminase as the autoantigen of coeliac disease. Nature Medicine 3:797-801.

Drago S., El Asmar R,, Di Pierro M., Clemente M.G., Sapone A., Thakar M., Iacono G., Carroccio A., D’Agate C., Not T. (2006) Gliadin, zonulin, and gut permeability: effect on celiac and nonceliac intestinal mucosa and intestinal cell lines. Scandnavian J Gastroenterology 41:408-419.

Dubnau, D. (1985) Induction of erm $C$ requires translation of the leader peptides. The EMBO Journal 4:533-537.

Fleckenstein, B. et al. (2002) Gliadin T-cell epitope selection by tissue transglutaminase in celiac disease: role of enzyme specificity and $\mathrm{pH}$ influence on the transamidation vs. deamidation reactions. J. Biological Chemistry 277:3410934116

Greco, L., et al. (2002) The first large population based twin study of coeliac disease. Gut. 50:624-628.

Gryczan TJ., Grandi G., Hahn J., Grandi R., Dudnau D. (1980) Conformational alteration of mRNA structure and the posttranscriptional regulation of erythromycin induced drug resistance. Nucleic acids research 8:6081-6097.

Hakenbeck R., Messer W. (1977) Activity of Murein Hydrolases in Synchronized Cultures of Escherichia coli. J Bacteriology 129:1239-1244.

Hausch, F., Shan, L., Santiago, N.A., Gray, G.M., Khosla, C. (2002) Intestinal digestive resistance of immunodominant gliadin peptides. American Journal of Physiology: Gastrointestinal and Liver Physiology. 283; 996-1003.

Heng NCK. Personal Communication.

Hourinouchi S., Weisblum B. (1982) Nucleotide sequence and functional map of $\mathrm{pE194}$, a plasmid that specifies inducible resistance to macrolide, lincosamide, and streptogramin type B antibiotics. J Bacteriology 150:804-814.

Hue KK., Bechhofer DH. (1991) Effect of ermC Leader Region Mutations on Induced mRNA Stability. J Bacteriology 173:3732-3740.

Kim Y.H., Han K.S., Oh S., You S., Kim S. (2005) Optimization of techniques for the transformation of Lactobacillus acidophilus strains by electroporation. Journal of Applied Microbiology. 99:167-174.

Kok J., van der Vossen JMBM., Venema G., (1984) Construction of Plasmid Cloning Vectors for Lactic Streptococci Which Also Replicate in Bacillus subtilis and Escherichia coli. Applied and Environmental Microbiology 48:726-731.

Lammers et al., (2008) Gliadin Induces an Increase in Intestinal Permeability and Zonulin Release by Binding to the Chemokine Receptor CXCR3. Gastroenterology 135:194-204.

Marteau, P., Minekus, M., Havenaar, R., Huis In’t Veld, J. (1997) Survival of lactic acid bacteria in a dynamic model of the stomach and small intestine: validation and the effects of bile. Journal of Dairy Science 80; 1031-1037.

Mayford M., Weisblum B. (1989) Conformational alterations in the ermC transcript in vivo during induction. The EMBO Journal 8:4307-4314. 
Molberg, O., McAdam, S.N., Korner, R., Qartsten, H., Kristiansen, C., Madsen, L., Fugger, L., Scott, H., Noren, O., Roepstorff, P., Ludnin, K.E.A., Sjostrom, H., Sollid, L.M., 1998. Tissue transglutaminase selectively modifies gliadin peptides that are recognised by gut-derived T-cells in celiac disease. Nat. Med. 4, 713-717.

Mukai, T., Kaneko, S., Matsumoto, M., Ohori, H. (2004) Binding of Bifidobacterium bifidum and Lactobacillus reuteri to the carbohydrate moieties of intestinal glycolipids recognized by peanut agglutinin. International Journal of Food Microbiology 90; 357-362.

Naidu, A. S., Bidlack, W.R., and Clemens, R.A. (1999). Probiotic spectra of lactic acid bacteria. Critical review in food science and nutrition. 39: 13-126.

Narita J., Ishida S., Okano K., Kimura S., Fukuda H., Kondo A., (2006) Improvement of protein production in lactic acid bacteria using 5'-untranslated leader sequence of slpA from Lactobacillus acidophilus. Improvement in protein production using UTLS. Applied Microbial Biotechnology 73: 366-373.

Picarelli A., Sabbatella L., Di Tola M., Gabrielli F., Greco R., Di Cello T., Mastracchio A., Anania MC. (2000) Celiac disease diagnosis in misdiagnosed children. Pediatric Research 48:590-592.

Polgar, L. The prolyl oligopeptidase family. (2002) Cellular and Molecular Life Sciences 59:349-362.

Rizzelo CG et al. (2007) Highly Efficient Gluten Degradation by Lactobacilli and Fungal Proteases during Food Processing: New Perspectives for Celiac Disease. Applied and Environmental Microbiology 73:4499-4507.

Rollan, G., Font de Valdez, G. (2001) The peptide hydrolase system of Lactobacillus reuteri. International Journal of Food Microbiology. 70; 303-307.

Ross, P., Morgan, S., Hill, C. (2002) Preservation and fermentation: past, present and future. International Journal of Food Microbiology 79(1-2); 3-16.

Ruben, C.F., Brandborg, L.L., Flick, A.L., et al. (1962) Biopsy studies on the pathogenesis of coeliac sprue in intestinal biopsy. In: Wolstenhome, G.E.W., Camera, M.P. (Eds.), London Ciba Foundation Study Book 14.

Saavedra, J. (2000) Probiotics and infectious diarrhea. The Journal of Gastroenterology 95(1).

Sanz, Y., Toldra, F. (2001) Purification and characterization of an X-prolyl-dipeptidyl peptidase from Lactobacillus sakei. Applied and Environment Microbiology 67(4); 1815-1820.

Servin, A. (2004) Antagonistic activities of lactobacilli and bifidobacteria against microbial pathogens. FEMS Microbiology Reviews 28; 405-440.

Shan, L., Marti, T., Sollid, L., Gray, G., Khosla, C. (2004) Comparative biochemical analysis of three bacterial prolyl endopeptidases: implications for coeliac sprue. Biochemistry Journal 383; 311-318.

Shan, L., Molberg, O., Parrot, I., Hausch, F., Filiz, F., Gray, G.M., Sollid, L.M., Koshla, C. (2002) Structural basis for gluten intolerance in coeliac sprue. Science 297; 2275-2279.

Shanahan, F. (2004) Probiotics in inflammatory bowel disease-therapeutic rationale and role. Advanced Drug Delivery Reviews 56; 809-818. 
Shivakumar AG., Hahn J., Grandi G., Kozlov Y., Dubnau D. (1980) Posttranscriptional regulation of an erythromycin resistance protein specified by plasmid pE194. PNAS 77:3903-3907.

Smit E., Pouwels PH. (2002) One Repeat of the Cell Wall Binding Domain Is Sufficient for Anchoring the Lactobacillus acidophilus Surface Layer Protein. Applied and Environmental Microbiology 186:4617-4619.

Sollid LM., Thorsby E. (1993) HLA susceptibility genes in coeliac disease: genetic mapping and role in pathogenesis. Gastroenterology 105:910- 22.

Stepniak D., et al. Highly efficient gluten degradation with a newly identified prolyl endoprotease: implications for celiac disease (2006). Am. J. Physiol. Gastrointest. Liver Physiol. 291:621-629.

Stiles, M.E., \& Holzapfel, W.H. (1997). Lactic acid bacteria of food and their current taxonomy. International Journal of Food Microbiology, 36(1), 1-29.

Tannock, G.W. (2004). A special fondness for Lactobacilli. Applied and Environmental Microbiology, 70(6), 3189-3194.

Thomas KE., Sapone A., Fasano A., Vogel, S. (2006) Gliadin Stimulation of Murine Macrophage Inflammatory Gene Expression and Intestinal Permeability Are MyD88-Dependent: Role of the Innate Immune Response in Celiac Disease. $J$ Immunology, 2512-2521.

Valeur, N., Engel P., Carbajal N., Connoly E., Ladefoged K. (2004) Colonization and Immunomodulation by Lactobacillus reuteri ATCC 55730 in the Human Gastrointestinal Tract. Applied and Environment Microbiology 70:1176-1181.

Varmanen P., Rantanen T., Palva A., Tynkkynen S. (1998) Cloning and Characterization of a Prolinase Gene (pepR) from Lactobacillus rhamnosus 64:1831-1836.

Watts T et al. (2005) Role of the intestinal tight junction modulator zonulin in the pathogenesis of type I diabetes in BB diabetic-prone rats. PNAS 102:2916-2921.

Xia J., Sollid, L.M., Khosla, C., (2005) Equilibrium and kinetic analysis of the unusual binding behavior of a highly immunogenic gluten peptide to HLA-DQ2. Biochemistry 44: 4442-4449.

Xu, M., Li, Y., Jie, L., Min, D., Liu, J. (2002) An X-prolyl dipeptidyl aminopeptidase from Lactococcus lactis: Cloning, expression in Escherichia coli, and application for removal of N-terminal Pro-Pro from recombinant proteins. Protein Expression and Purification 24; 530-538.

Yeh C., Huang X., Sue C. (2008) Functional Secretion of a Type 1 Antifreeze Protein Analogue by Optimization of Promoter, Signal Peptide, Prosequence, and Terminator in Lactococcus lactis. Journal of Agricultural and Food Chemistry 56:8442-8450.

Yuksel GU., Steele JL. (1996) DNA sequence analysis, expression, distribution and the physiological role of the Xaa-prolyldipeptidyl aminopeptidase gene from Lactobacillus helveticus CNRZ32. Applied Microbial Biotechnology 44:766-773. 